\title{
Beam monitor system for an x-ray free electron laser and compact laser
}

\author{
Y. Otake, ${ }^{1,2}$ H. Maesaka, ${ }^{1,2}$ S. Matsubara, ${ }^{2}$ S. Inoue, ${ }^{5}$ K. Yanagida, ${ }^{3}$ H. Ego, ${ }^{1,2,3}$ C. Kondo, ${ }^{1,2}$ T. Sakurai, ${ }^{1,2}$ \\ T. Matsumoto, ${ }^{4}$ and H. Tomizawa ${ }^{1,2}$ \\ ${ }^{1}$ RIKEN, SPring-8 Center, XFEL Research and Development Division, \\ 1-1-1 Kouto, Sayo-cho, Sayo-gun, Hyogo, 679-5148, Japan \\ ${ }^{2}$ JASRI, XFEL Division, 1-1-1 Kouto, Sayo-cho, Sayo-gun, Hyogo, 679-5198, Japan \\ ${ }^{3}$ JASRI, Accelerator Division, 1-1-1 Kouto, Sayo-cho, Sayo-gun, Hyogo, 679-5198, Japan \\ ${ }^{4}$ JASRI, Control and Computing Division, 1-1-1 Kouto, Sayo-cho, Sayo-gun, Hyogo, 679-5198, Japan \\ ${ }^{5}$ SPring-8 Service Co. Ltd. 1-20-5 Kouto, Shingu-cho, Tatsuno-shi, Hyogo, 679-5165, Japan
}

(Received 15 August 2012; published 23 April 2013)

\begin{abstract}
A beam-monitor system for XFEL/SPring 8, "SACLA," has been constructed. In order to maintain a stable self-amplified spontaneous emission (SASE) interaction, the straightness and overlap of the axes to within $3 \mu \mathrm{m}$ between the electron beams and the radiated $\mathrm{x}$ rays for an undulator section of about $100 \mathrm{~m}$ length is necessary. This straightness means relative alignment to an experimental target sample. Furthermore, a temporal stability of $30 \mathrm{fs}$ in order to maintain a constant peak beam current is also necessary to conduct stable SASE lasing. The monitor system was developed to satisfy these spatial and temporal stability and resolution criteria. The system comprises spatial monitors, such as cavity-type beam-position monitors and screen monitors, as well as temporal measurement instruments, such as current monitors, waveguide spectrometers, coherent synchrotron-radiation detectors, a streak camera, and an rf deflector. Commissioning of SACLA started from March 2011, and the monitors performed sufficient roles to tune the beams for lasing. The achieved overall performances of the system, including data acquisition, are: the beam position monitor has a spatial resolution of $600 \mathrm{~nm}$ in rms; the bunchlength monitors show ability to observe bunch lengths from $1 \mathrm{~ns}$ in an injector with velocity bunching to less than $30 \mathrm{fs}$ after three-stage bunch compressors. The less than $3 \mu \mathrm{m}$ spatial resolution of the screen monitor was also confirmed during practical beam operation. Owing to these fulfilled performances, such as the high spatial and temporal resolutions, stable lasing of SACLA has been achieved.
\end{abstract}

DOI: 10.1103/PhysRevSTAB.16.042802

PACS numbers: $41.60 . \mathrm{Cr}$

\section{INTRODUCTION}

The x-ray free electron laser (XFEL), "SACLA (SPring-8 angstrom compact laser)," as shown in Fig. 1, has been constructed [1]. It comprises an $8-\mathrm{GeV}$ linear accelerator, which is $400 \mathrm{~m}$ long, and 18 in-vacuum undulators, each being $5 \mathrm{~m}$ long. The accelerator consists of an injector including 238, 476, and $1428 \mathrm{MHz}$ multi-sub-harmonic bunchers, and a main accelerator using $2856 \mathrm{MHz}$ (S-band) and $5712 \mathrm{MHz}$ (C-band) traveling-wave accelerating structures. The XFEL is expected to open up new experimental methods of science, such as single-shot and particle $\mathrm{x}$-ray diffraction imaging [2]. We succeeded in the development and construction of SACLA, which lased at a wavelength of $0.063 \mathrm{~nm}$ in July 2011 [1]. In order to maintain stable $\mathrm{x}$-ray laser using the process of selfamplified spontaneous emission (SASE), SACLA is demanded to exhibit extreme spatial and temporal stability of its accelerated electron beam.

Published by the American Physical Society under the terms of the Creative Commons Attribution 3.0 License. Further distribution of this work must maintain attribution to the author(s) and the published article's title, journal citation, and DOI.
The necessity for this stability is explained as follows [3-5]. The power evolution of a free electron laser (FEL) is according to the Pierce gain parameter, $\rho$, which is expressed by [4]

$$
\begin{gathered}
\rho=\left(\frac{\gamma \lambda^{2} r_{e} n_{e}}{8 \pi} F(K)\right), \\
F(K)=\frac{K^{2}}{\left(1+\frac{K^{2}}{2}\right)^{2}}\left\{J_{0}\left(\frac{\frac{K^{2}}{4}}{1+\frac{K^{2}}{4}}\right)-J_{1}\left(\frac{\frac{K^{2}}{4}}{1+\frac{K^{2}}{4}}\right)\right\}^{2}, \\
\lambda=\frac{\lambda_{u}}{2 \gamma^{2}}\left(1+\frac{K^{2}}{2}\right),
\end{gathered}
$$

where $\gamma$ is the relativistic energy, $\lambda$ is the radiated wavelength of FEL, $r_{e}$ is the classic radius of an electron, $n_{e}$ is the electron density (population), and $K$ is the deflection parameter; $J_{0}$ and $J_{1}$ are Bessel functions of the 0 th and 1 st order, respectively, and $\lambda_{u}$ is the periodic length of the permanent magnet cells of the undulator.

In the case of the spatial stability, the electron beam should be precisely aligned with its radiated $x$ rays for their stable interaction amplifying an $\mathrm{x}$-ray laser. This laser should also be relatively and periodically aligned to an experimental target sample, and the laser pointing must 
DCT: 14

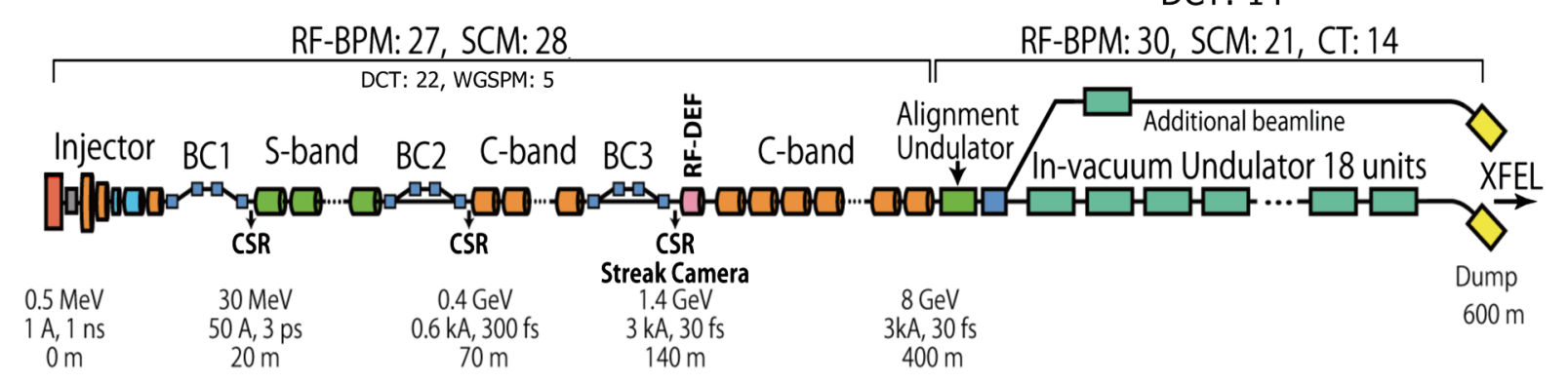

FIG. 1. SACLA machine layout and beam monitors.

keep to the target by mitigating effects from any environmental perturbations, such as ground subsidence. This overlap relation is expressed by

$$
\rho=\frac{O F^{1 / 3}}{\gamma_{0}}\left(\frac{\omega_{b} \frac{K}{\sqrt{2}}}{4 c k_{u}}\right)^{2 / 3}
$$

between $\rho$ and an overlap factor, $O F=\sigma_{b} / \sigma_{r}$ [5], where $\sigma_{b}$ is the rms beam size, $\sigma_{r}$ is the rms radiation size, $\gamma_{0}$ is the electron rest energy of the $Z$ direction (moving direction), $k_{u}$ is the wave number of $\lambda_{u}$, and $\omega_{b}$ is the plasma frequency of the electron beam. Since $O F$ directly affects $\rho$ in Eq. (3), the electron beam and its radiation along an undulator section are tightly aligned. Because the overlap between them determines the strength of nonlinear interaction in order to form electron microbunches. Single-kick errors [6] due to position misalignments of the accelerator components decrease $O F$. For example, these misalignments are caused by position differences among the magnetic centers of quadrupole magnets and the error dipole field of the undulators. In the case of SACLA, this overlap should be to within $4 \mu \mathrm{m}$ through an undulator section, if we allow degradation of the FEL power to be half of the ideal case [6]. Therefore, beam monitors for the SACLA accelerator should have a spatial position resolution of less than $1 \mu \mathrm{m}$ to secure the required overlap.

Another demand on the spatial resolution to the monitors is to measure the emittance of the electron beam, since $\rho$ is dependent on the effective energy spread, $(\delta \gamma / \gamma)_{\text {eff }}$, including the emittance, $\varepsilon$, as expressed by

$$
\rho \gg\left(\frac{\delta \gamma}{\gamma}\right)_{\mathrm{eff}}=\sqrt{\left(\frac{\delta \gamma}{\gamma_{0}}\right)^{2}+\left(\gamma_{z 0}^{2} \frac{\varepsilon}{\sigma_{b}^{2}}\right)^{2}},
$$

where $\gamma_{z 0}$ is the rest energy in the $z$ direction and $\sigma_{b}$ is the beam size in rms. When FEL amplification is developed, the effective energy spread, $(\delta \gamma / \gamma)_{\text {eff }}$, must be much smaller than the Pierce gain parameter, $\rho$. Because $\rho$ in Eq. (1) directly reflects $\gamma$ and $n_{e}$. The electron density, $n_{e}=q_{e} / \sigma_{e}$, inversely decreases according to an increase of the emittance, $\varepsilon=\left(\sigma_{e}\right)^{2} / \beta$. In this case, $q_{e}$ is the electron charge amount, $\sigma_{e}$ is the rms beam size, and $\beta$ is the betatron wavelength. Therefore, $\varepsilon$ must be small to preserve $O F$ between the electron beam and its emitted radiation [5]. $\mathrm{A} \mathrm{CeB}_{6}$ thermionic electron gun with a $500 \mathrm{kV}$ high-voltage pulse with a $2 \mu$ s width (FWHM) presently emits an electron beam with an emittance of less than $1 \pi \mathrm{mm}$ mrad. Even though the electron beam is accelerated through the long accelerator, and the bunch length of the beam is compressed at three bunch compressors (BC), as mentioned below, the emittance should be maintained to obtain a proper FEL amplification gain, because of the relation of Eqs. (1) and (4). Therefore, measurements of the emittance by the $Q$ scan method [7] with our monitors are crucial for beam tuning.

The electron bunch is compressed from $1 \mathrm{~ns}$ to $30 \mathrm{fs}$ along the SACLA accelerator in order to form the $3 \mathrm{kA}$ peak current. This process is achieved by using the velocity-bunching process in multi-sub-harmonic bunchers (SHB) of the injector [8] and the magnetic bunching process in the $\mathrm{BC}$ using a chicane comprising four bending magnets. For temporal stability in order to maintain the FEL intensity to within $10 \%$, an approximately $30 \mathrm{fs}$ (FWHM) bunch length and a bunch charge fluctuation of $10 \%$, which is formed by the above-mentioned bunching process, should be tightly maintained [9]. This demand of temporal stability is understandable from the relation between the $n_{e}$ and $\rho$ of Eq. (1). Since the electron bunch is compressed to $30 \mathrm{fs}$, a temporal resolution of less than $30 \mathrm{fs}$ is also required of a beam monitor for observing the beam characteristics along the accelerator to form the peak current and a bunch length of $30 \mathrm{fs}$.

\section{BEAM MONITORS}

\section{A. Outline of beam monitors}

We have developed and constructed beam monitors in order to observe the spatial and temporal characteristics of the beams, as illustrated in Fig. 1, and in order to satisfy the above-mentioned demands; they are already and effectively used for beam tuning in SACLA. The design concepts of these monitors are to reduce any vibration by firm and stable mechanical structures and to reduce noise and characteristic drift in detection circuits and a data acquisition (DAQ) system in order to secure detection accuracy. On the other hand, the precise mechanical accuracy for maintaining the straightness of the accelerator and the 
undulator line by alignment is crucial for SACLA. Therefore, accurate machining in the production of the monitors in order to realize the alignment is also one of the concepts.

For spatial observations of electron beams in order to realize the above-mentioned demands, such as the position and the profile resolutions, 57 cavity-type beam-position monitors (RF-BPM) [10] and 49 high-resolution screen monitors (SCM) [11] were installed in SACLA. The RFBPM detects a beam-induced field, and the SCM observes optical transition radiation (OTR) [12] and scintillation of Ce:YAG. These demanded resolutions are less than $1 \mu \mathrm{m}$ for the BPM and $4 \mu \mathrm{m}$ for the SCM, respectively.

In order to observe the electron bunch lengths along the bunching process, as temporal measurements, two fastdifferential current transformers (DCT) [13] and five waveguide spectrometers (WGSPM) [14] are installed for the beginning part of the injector of SACLA. Furthermore, three coherent synchrotron-radiation monitors (CSRM) [15] for the three BCs, an OTR bunch-length monitor, and a HEM11-mode C-band rf beam deflector (RFDEF) [16] were installed downstream after the injector. Thirtythree DCTs in total used to measure the charge amount of the beam were also installed in other accelerator parts without the beginning of the injector. Since the DCT has a pulse response of $200 \mathrm{ps}$ (10\%-90\% pulse rise time), it can measure the bunch length around the injector. The WGSPM provides ability to observe any relative bunchlength variation by a velocity-bunching process in the injector. The OTR bunch-length monitor uses a streak camera (FESCA-200, Hamamatsu Photonics Co. Ltd.). The rf deflector is operated at $5712 \mathrm{MHz}$ in order to observe the final longitudinal bunch structure after the BC3. Table I summarizes the numbers and some features of the above-mentioned monitors.

The beam-monitor system, the individual beam monitors, the detection circuit, the DAQ system, and the performances of the monitors, such as the spatial and temporal resolutions, are mainly described in this paper.

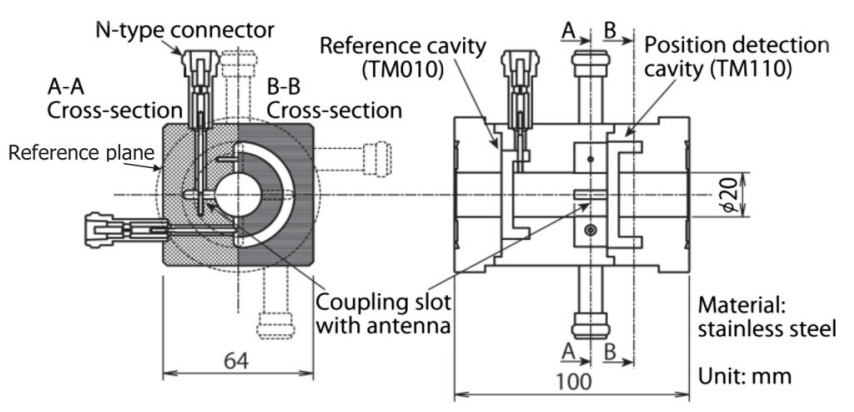

FIG. 2. Cross-sectional schematics of the BPM.

\section{B. Spatial beam monitors}

\section{BPM}

The BPM, as depicted in Fig. 2, comprises a position detection cavity with a TM110 mode and a reference cavity with a TM010 mode, both operated at a resonant frequency of $4760 \mathrm{MHz}$. The reason why the $4760 \mathrm{MHz}$ frequency was chosen is to reduce any effect due to dark current generated by the $\mathrm{C}$-band accelerating structures operated at $5712 \mathrm{MHz}$. The position detection cavity has four coupling slots with antennas, two for the $X$ direction and the other two for the $Y$ direction. The material of a cavity body is stainless steel in order to make a low-quality $(Q)$ factor of about 500. This $Q$ factor decreases any perturbation effect due to an environmental temperature change to the detection sensitivity, as well as obtaining a fast pulse response. A reference plane for alignment, shown in Fig. 2, is provided on the outer surface of the BPM body. The three-dimensional position of the reference plane from the mechanical center of the position detection cavity is measured with an accuracy of within $\pm 10 \mu \mathrm{m}$.

Detection sensitivity measurements for the BPMs were carried out by using the detection circuit described below and a motorized stage under the BPM. This stage is provided for the relative alignment of the undulator line of a length of $700 \mathrm{~m}$ and the position sensitivity calibration for the BPM. Figure 3 shows position detection sensitivity

TABLE I. Kinds and numbers of beam monitors for SACLA.

BPM (cavity beam position monitor)

SCM (screen monitor)

DCT (differential current transformers)

WGSPM (waveguide spectrometer)

CSRM (coherent synchrotron radiation monitor)

OTR bunch-length monitor using the streak camera

RFDEF (rf deflector)
Total 57

Total 49

3 desmarquests

(Desmarquest Co., $\mathrm{Cr}_{2} \mathrm{O}_{3}-0.5 \%$ doped $\mathrm{Al}_{2} \mathrm{O}_{3}$, for the injector) 46 stainless (OTR) or scintillation (Ce:YAG) screens (for regular section)

Total 36

1 single-end output

35 differential output

5 in the injector

3 at the $3 \mathrm{BCs}$

2 at the $\mathrm{BC} 1$ and $\mathrm{BC} 2$

1 after the $\mathrm{BC} 3$ 


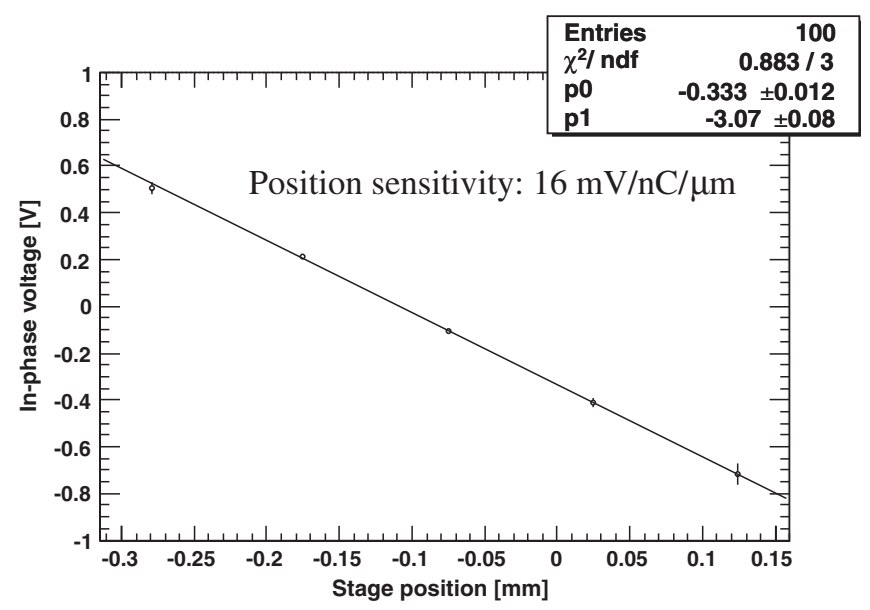

FIG. 3. Position detection sensitivity of the cavity-type BPM. The sensitivity is $16 \mathrm{mV} / \mathrm{nC} / \mu \mathrm{m}$.

curves that were measured at the SACLA accelerator, while the $X$ stage attached under the BPM was moved in accordance with the horizontal-axis values of the graph. From the data of the graph, we calculated the position sensitivity, being $16 \mathrm{mV} / \mathrm{nC} / \mu \mathrm{m}$. The position dependence of the $Y$ direction is almost the same as that of the $X$ direction. The coupling between both the $X$ and $Y$ detection cavities are negligibly small, being less than $-30 \mathrm{~dB}$.

A detection circuit for the BPM uses the in-phase quadrature (IQ) method, as shown in Fig. 4. The noise level of the circuit is about $1 \times 10^{-4} \mathrm{~V}$ in rms. This noise level corresponds to a BPM position resolution of $0.2 \mu \mathrm{m}$, when the measurement conditions of an accelerated electron charge amount of $0.3 \mathrm{nC}$ and the $\pm 1 \mathrm{~V}$ maximum input voltage of the DAQ system are set up. The detected signal is digitized and acquired with a VERSA module Eurocard bus (VME) 16-bits analog-to-digital converter (ADC), as shown in Fig. 5, for the computer control system. The effective dynamic range to be not affected by noise is 13-14 bits, which corresponds to within a $1 \times 10^{-4}$ resolution. Therefore, the noise level of the detector and the ADC dynamic range are sufficient to achieve a submicron meter position resolution.

After mass production of the BPMs, they were installed into the SACLA accelerator tunnel and an undulator beam

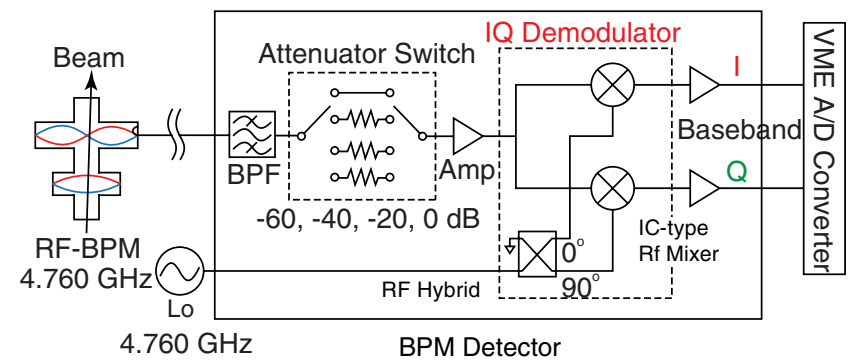

FIG. 4. Configuration of the BPM detector using the IQ demodulator.

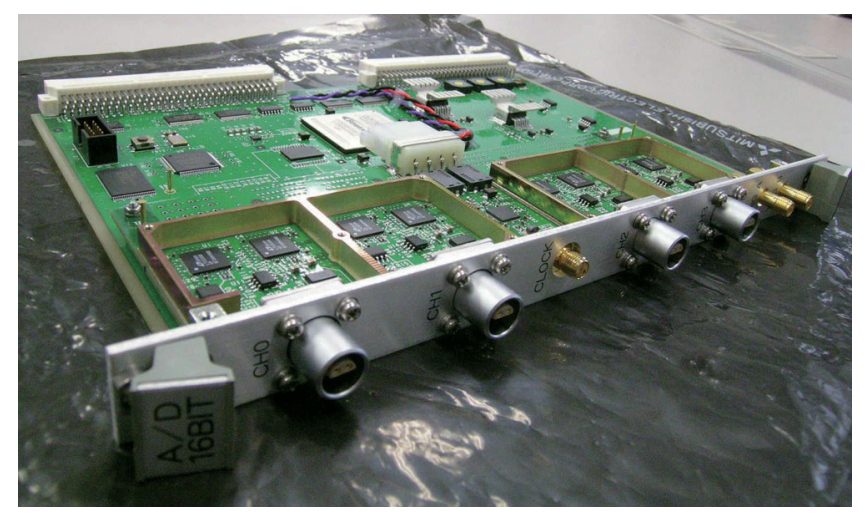

FIG. 5. VME ADC module (16 bits) driven by a $238 \mathrm{MHz}$ rf signal.

line room. Their performances were evaluated, when the beam commissioning of SACLA was conducted. The position resolutions of the 20 BPMs along the undulator beam line were precisely checked, because the resolutions were directly associated with the performance of the beam line tuning for SASE amplification. The position resolution of a BPM was evaluated from the difference value between a measured position and an estimated one from other neighboring BPMs. We analyzed the resolutions of the 20 BPMs along the undulator beam line. In order to estimate the beam position at a given RF-BPM, the data of the other 19 RF-BPMs were used. The beam positions of the given RFBPM to individual beam shots were estimated by the leastsquares method. In this analysis, constraints coming from transfer matrices were imposed on a beam orbit. The positions and slopes of the beams at the given RF-BPM were determined so as to reproduce the other BPM data. By using an electron beam with a bunch charge of $0.1 \mathrm{nC}$ and a beam energy of $7 \mathrm{GeV}$, we analyzed the position resolutions of the RF-BPMs on the undulator beam line. Figure 6 shows the evaluated position resolutions of the 20 BPMs. These resolutions are less than $0.6 \mu \mathrm{m}$ in rms.

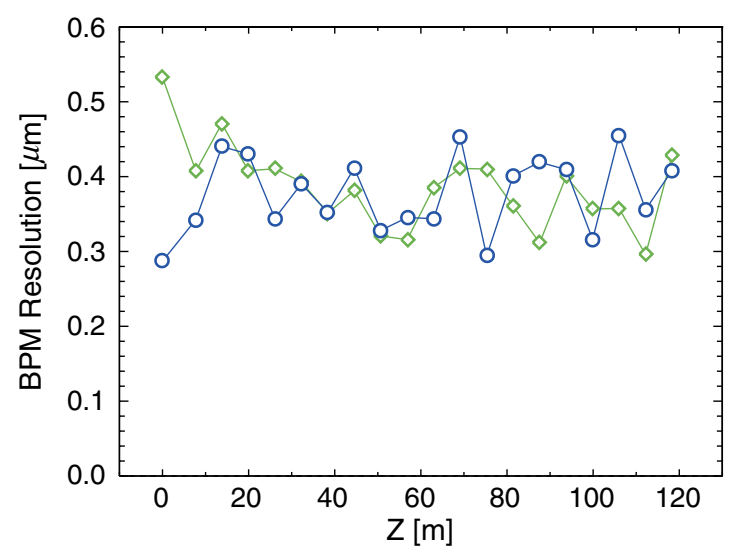

FIG. 6. Position resolution data along the 20 BPMs in the undulator beam line. The blue circles show the $X$ resolutions and the green circles are the $Y$ resolutions. The resolutions are less than $0.6 \mu \mathrm{m}$ (STD). 


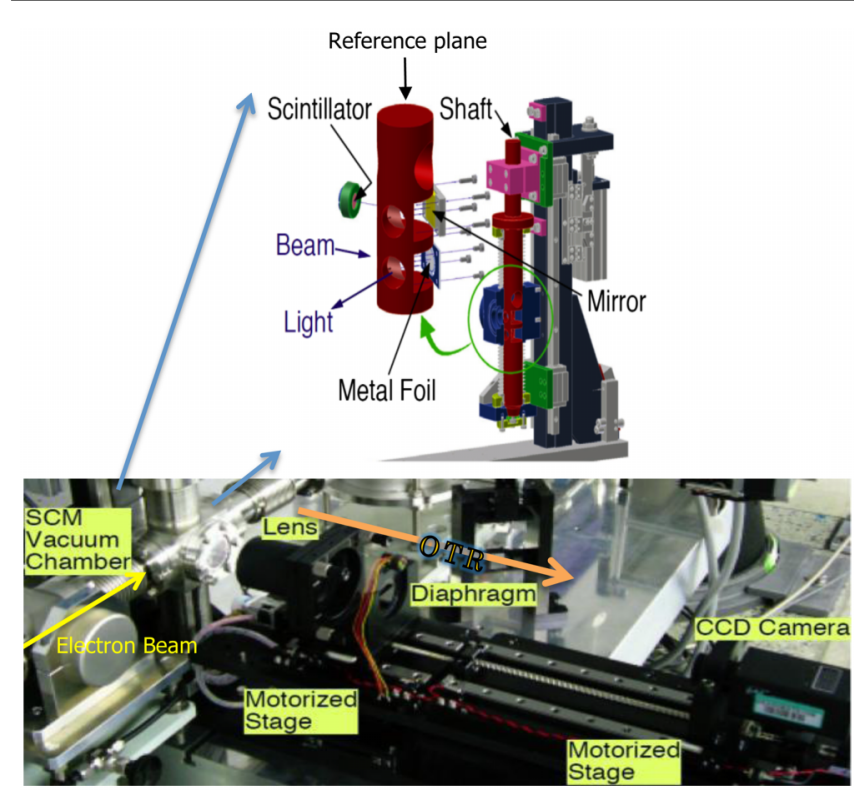

FIG. 7. Mechanical structure in the vacuum chamber and the optical system for the SCM.

\section{SCM}

The SCM, as shown in Fig. 7, comprises a vacuum chamber, an in-vacuum screen of stainless-steel foil in order to radiate OTR, an alumina-fluorescent screen (Desmarquest) or a Ce:YAG screen with a $100 \mu \mathrm{m}$ thickness in order to emit scintillation light, focusing lenses with three groups and four pieces, and a CCD camera system. A pneumatic cylinder drives the screen. The reference plane for alignment is provided on the outer surface of the SCM body, as shown in Fig. 7. The threedimensional position of the reference plane from the mechanical center of the screen is measured in accuracy to within $\pm 10 \mu \mathrm{m}$.

The stainless-steel screen consists of ten foil targets with a $100 \mu \mathrm{m}$ thickness, each, which is joined by a diffusion bonding method. One target foil having a mirror surface of $40 \mathrm{~nm}$ roughness is used to take a beam image; the other foils have beam holes where the mirror surface is placed. This method makes one target with $100 \mu \mathrm{m}$ thickness and a firm frame with $1 \mathrm{~mm}$ thickness, as depicted in Fig. 8.

The lenses are placed near the screen with the distance between the front lens and the screen surface being $100 \mathrm{~mm}$; the lenses have a large aperture of 2 inches. This optical-geometrical structure is effective to obtain a wide numerical aperture. The simulated spatial resolution of the structure was $2.5 \mu \mathrm{m}$. After this numerical simulation, a trial SCM was produced. The spatial resolution of the optical system of the trial SCM was evaluated by observing a grid-distortion target, as shown in Fig. 9. The optical spatial resolution was estimated from contrast data of a target image taken by the observation. We defined the resolution as being a differentiated value of the contrast data at the edge of a circle on the target. The estimated

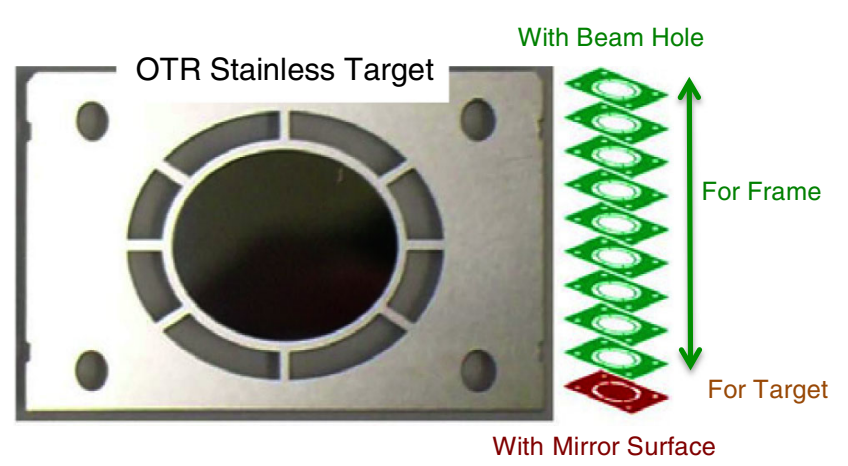

FIG. 8. Stainless-steel OTR screen (a $100 \mu \mathrm{m}$ thickness). Ten foil targets with a $100 \mu \mathrm{m}$ thickness, each of which are joined by the diffusion bonding method. One target has a mirror surface of a $40 \mathrm{~nm}$ roughness to take a beam image, and the other to make a firm frame has a beam hole.

spatial resolution was about $2.5 \mu \mathrm{m}$, which is consistent with the simulated value.

After this resolution evaluation, a trial SCM was installed in the SCSS test accelerator for a test experiment. The observed images of the SCM in the experiment were taken, as shown in Fig. 10. The beam images, which were horizontally focused by a quadrupole magnet on the target, were both taken by an OTR and a Ce:YAG target with beam conditions of $250 \mathrm{MeV}$ energy and a $0.3 \mathrm{nC}$ charge

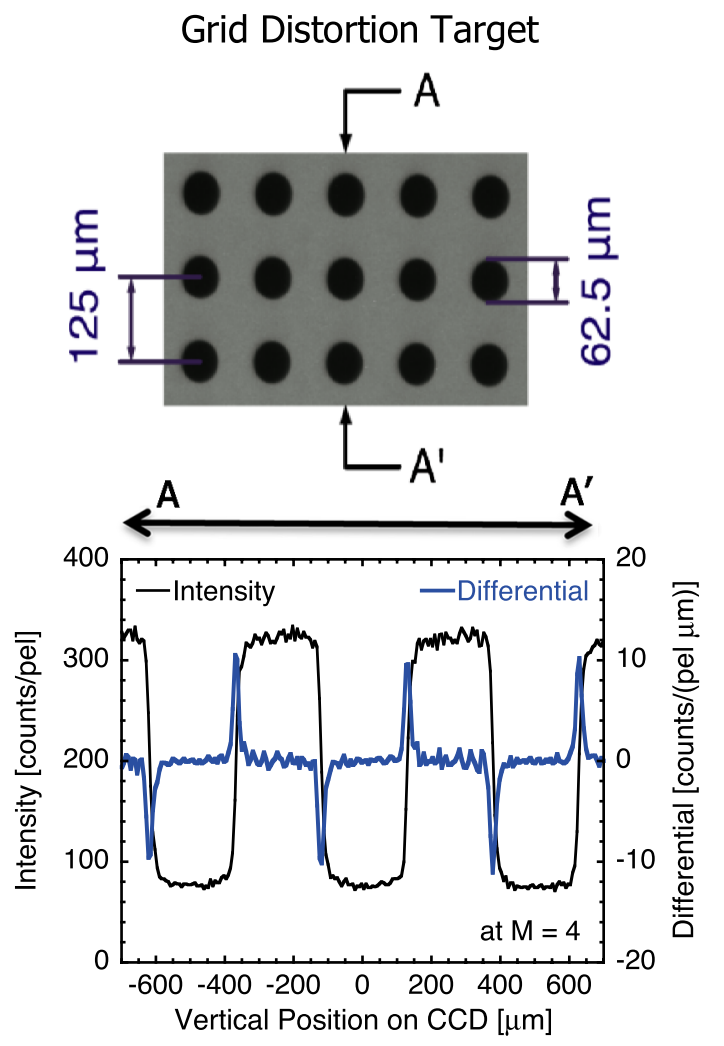

FIG. 9. Grid-distortion target and contrast data (black line) of the target image along the line from $\mathrm{A}$ to $\mathrm{A}^{\prime}$. The differentiation data (blue line) are also depicted. 


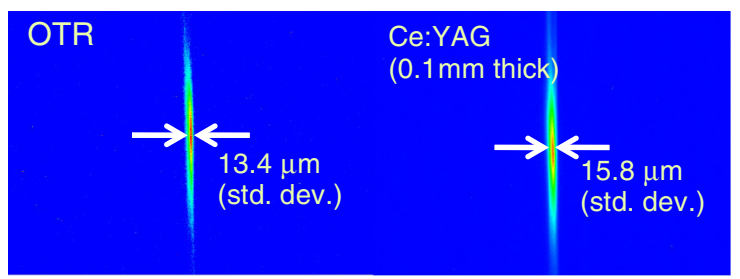

FIG. 10. Observed beam images of the SCM in an experiment at the SCSS test accelerator. (Left) The case of the OTR stainless-steel screen. (Right) The Ce:YAG fluorescence screen.

amount. The measured horizontal beam sizes were $13.5 \mu \mathrm{m}$ in the OTR case and $15.5 \mu \mathrm{m}$ in the Ce:YAG case, respectively. Both data strongly suggested realization of the above-mentioned spatial resolution.

We then mass produced $49 \mathrm{SCMs}$, and installed them in SACLA's tunnel. The SCM nicely worked upstream of the accelerator up to the $\mathrm{BC} 3$, when beam commissioning for SACLA was carried out. Coherent transition radiation (COTR) unfortunately occurred downstream of $\mathrm{BC} 3$, when the peak beam current became more than several kiloamperes. We then exchanged the screen material from stainless steel to Ce:YAG with an optical mask to reduce the COTR effect at an important point for beam tuning. The detailed story of the COTR will be discussed in another paper, which Dr. Matsubara is preparing.

We finally obtained a beam emittance measured by using our developed SCM and the $Q$ scan method at just the exit of $\mathrm{BC} 3$, as shown in Fig. 11, where the beam energy was $1.4 \mathrm{GeV}$. The $\beta$ function at the SCM was $55 \mathrm{~m}$, when the $K L$ value of the $Q$ magnet was zero. The normalized emittance value was measured to be $1.1 \pi \mathrm{mm}$ mrad. This fact is indirect evidence about the resolution of our SCM. Furthermore, the spatial resolution of the SCM was evaluated by comparing the observed center-mass positions of the beams between the BPM

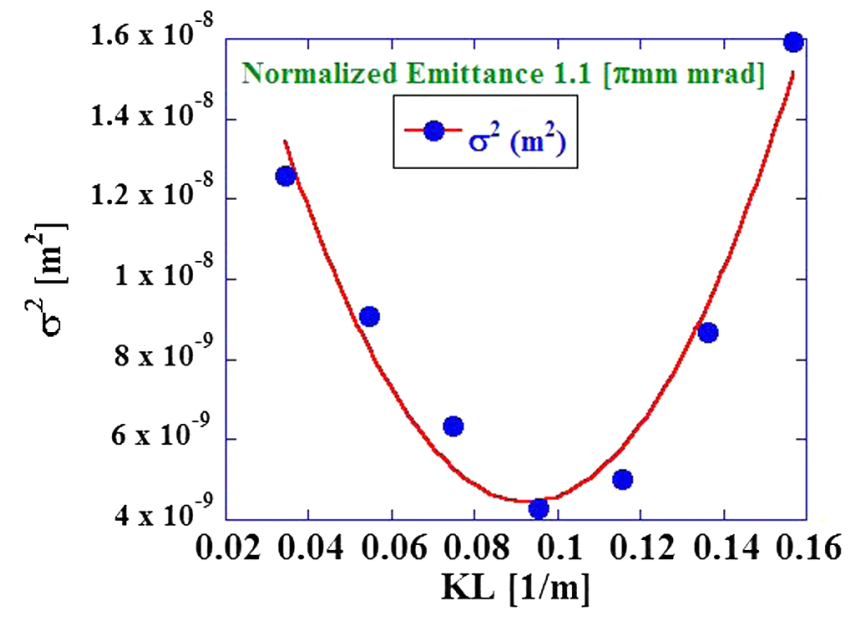

FIG. 11. Beam emittance measured by the $Q$-scan method at the position after BC3. The measured normalized emittance is $1.1 \pi \mathrm{mm}$ mrad. and SCM in the undulator beam line. This evaluation is discussed later in Sec. III.

\section{Temporal beam monitors \\ 1. DCT}

The fast DCT, as shown in Fig. 12, was developed to measure the beam current and the bunch length around the injector. The DCT has a function to reduce any commonmode noise by differential outputs. It comprises four outputs with a one-turn coil each, a circular Finemet core (Hitachi Metal, Ltd.), and an aluminum enclosure having an outer boxlike shape and an inner pillboxlike space. In order to not make the inner shape work as a resonant cavity, a resistive material (more than $3 \times 10^{-12} \Omega \mathrm{m}^{2}$ ) with a layered structure using nickel and chromium was coated on the inner surface of the cylinder. This coat has the function to dampen any beam-induced signal. One pair of coils was oppositely placed to each other along the horizontal axis, and the other pair was also oppositely placed along the vertical axis. The coil winding directions in the pair were set to obtain output signals with positive and negative signs, respectively. By subtracting the negative signal from the positive one in the pair, we can remove any common-mode noise. Moreover, the beam-position dependence is eliminated by averaging or summing the signals from all four output ports in order to obtain the total amount of beam charge.

Figure 13 shows the typical temporal beam wave forms (bunch shape) observed with the DCT in the injector, and Fig. 14 depicts an observed function in order to reduce common-mode noise. The rise time of the pulse outputted from the DCT was 200 ps (10\%-90\%). This value was measured with $19 \mathrm{GHz}$ cables and a $13 \mathrm{GHz}$ oscilloscope. This oscilloscope had a pulse rise time response of less than $80 \mathrm{ps}$ and a temporal resolution of less than $1 \mathrm{ps}$. This temporal resolution was provided by the time jitter

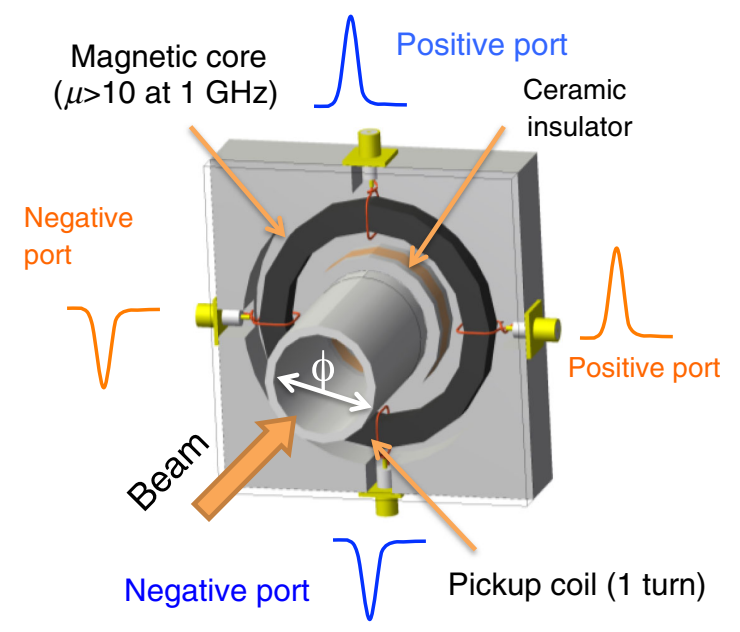

FIG. 12. Schematic drawing with the inside structure of the DCT. 


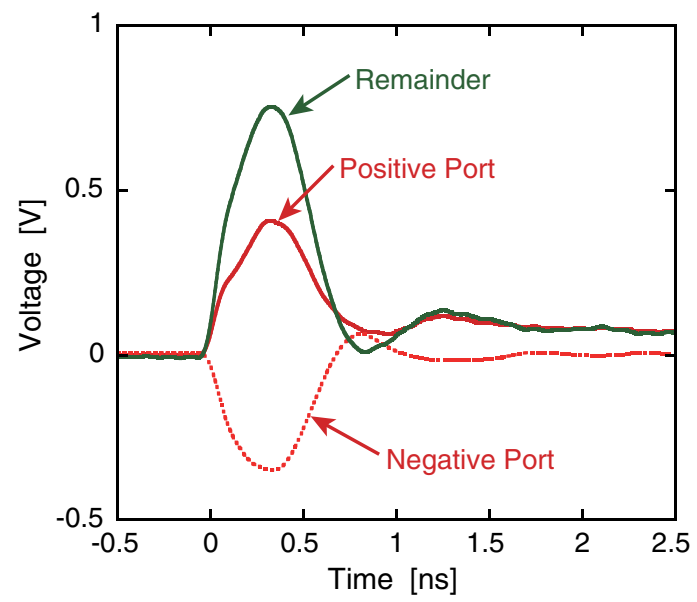

FIG. 13. Typical beam wave forms observed with the DCT in the injector. From up to down, the first (green line) is the subtracting signal between the signals from the positive and negative ports of the DCT in the horizontal. The second (red solid line) is the signal from the positive port and the third (red dotted line) is from the negative port.

measurement function of the oscilloscope. The detection sensitivity line, which is dependent on the charge amount, is shown in Fig. 15. The output voltage in the figure was measured as a function of the charge amount of the electron beam. This charge amount was measured with a current transformer (CT) made by Bergoz instrumentation [17], which had a known calibration factor provided by the company. This CT was also calibrated with a Faraday cup. Both the company-provided calibration factor and the

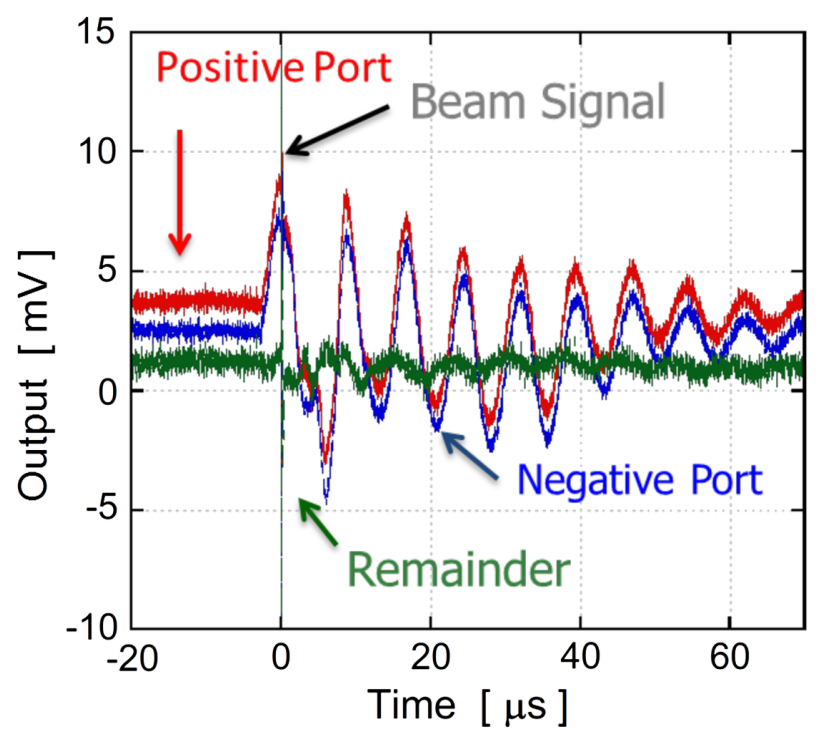

FIG. 14. Function in order to reduce any common-mode noise. The upper signal (red line) is from the positive port of the DCT in the horizontal. The second (blue line) is from the negative port. The third (green line) is the subtracting signal between the positive and negative signals.

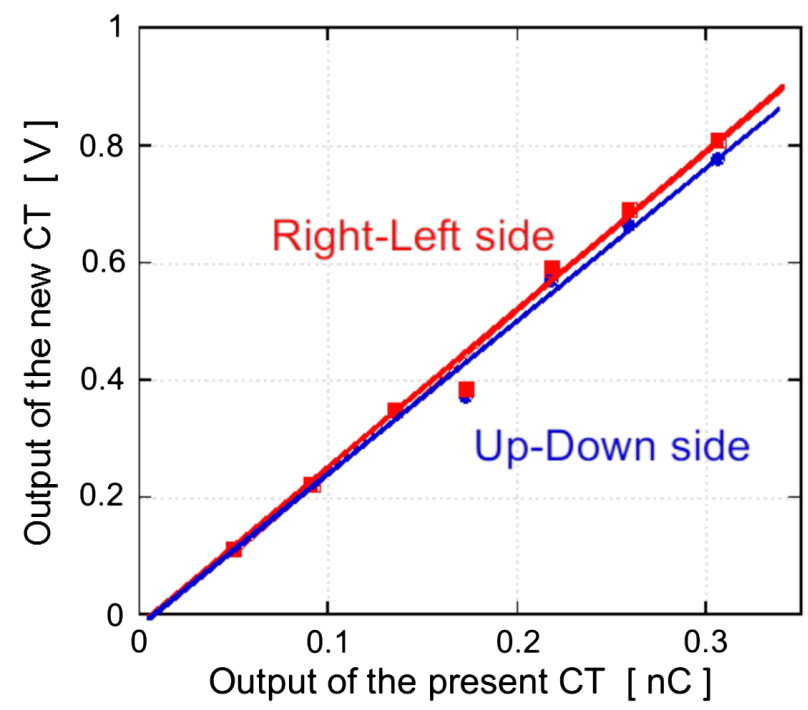

FIG. 15. Calibration of the DCT charge sensitivity. The output voltage was measured, while the charge amount of the electron beam was changed. This charge amount was measured with the CT made by Bergoz, which was calibrated with a Faraday cup. The red squares (right-left) show the subtracted data of the horizontal two ports and the blue circle (up-down) is the subtracted data of the vertical two-ports.

measured calibration factor with the Faraday cup were consistent. Because of the common-mode rejection function, the sensitivity is very high. Therefore, a dark current of about $1 \mathrm{pC}$ from a C-band accelerator was measured by the DCT.

On the other hand, the rough timing of the beam-arrival time can also be measured with the DCT, because of its fast pulse response. The above-mentioned 200 ps rise time is sufficient to observe a bunch length around a place just after the $238 \mathrm{MHz}$ SHB. To monitor the beam arrival timing drift at the $476 \mathrm{MHz}$ booster cavity, we measured the time-of-flight (TOF) between positions after the SHB and the booster cavities by using a setup in the injector, as shown in Fig. 16. Figure 17 expresses a change of the TOF, while the high-voltage value of the electron gun was changed. This result clearly shows that the DCT has a sufficient temporal resolution of less than 3 ps to measure the TOF variation by the velocity-bunching process.

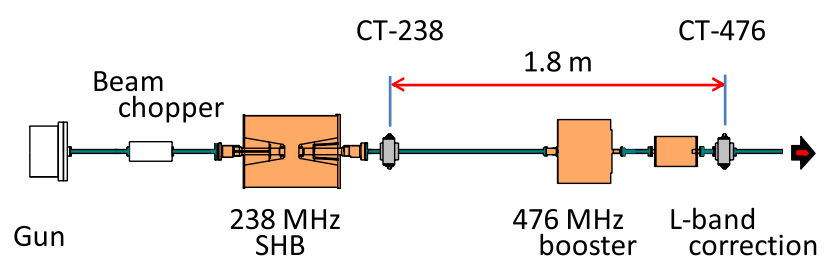

FIG. 16. Layout of the injector beginning part in SACLA including the beam chopper, the $238 \mathrm{MHz}$ SHB cavity, the $476 \mathrm{MHz}$ booster cavity, the L-band correction cavity and the DCTs of just after both cavities. 


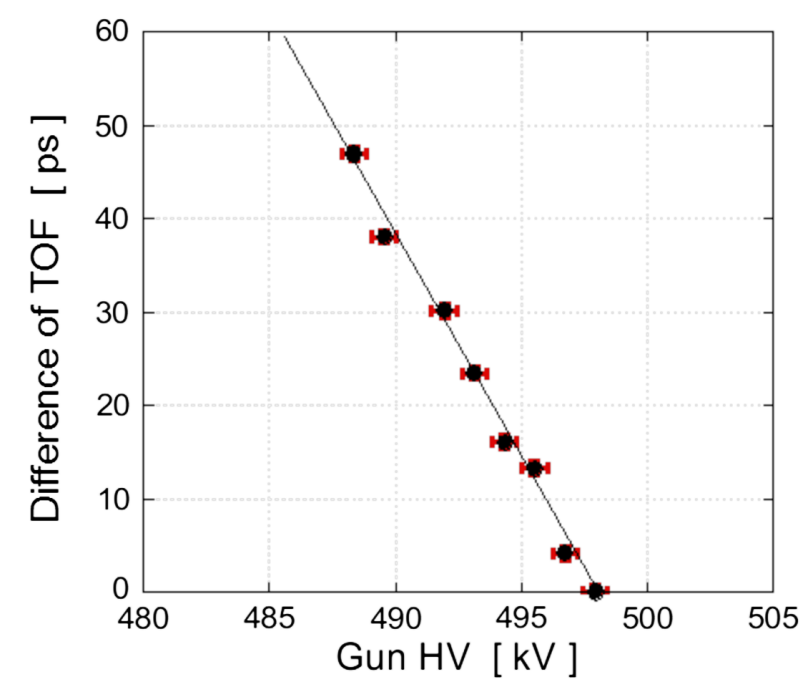

FIG. 17. Variation of the TOF measured by DCTs in the injector part of SACLA. The TOF change is plotted, while the electron-gun high voltage (HV) was changed. In this graph, the TOF value is normalized at 0 , when the high voltage (HV) of the gun is $498 \mathrm{kV}$. The horizontal and vertical error bars show fluctuation values in rms at individual points.

\section{Waveguide spectrometer}

When an electron beam hits the target of the SCM, such as Ce:YAG, coherent transition radiation (CTR) [18] is emitted from the surface of the target. The intensity of this radiation is enhanced around an optical wavelength corresponding to its pulse length. The CTR energy, $E(\omega)$, is expressed by an equation [18] as

$$
E(\omega)=2 \pi E_{t} N^{2}[\rho(\omega)]^{2},
$$

by using a population-weighted Fourier transformation of the bunch shape, $\rho(\omega)$, where $E_{t}$ is the total spectral radiation energy from a single electron, $N$ is the electron population, and $\omega$ is the angular frequency. Therefore, this radiation includes pulse-length information of the electron beam, and a wide frequency spectrum information. The CTR intensity is changed to be proportional to a change of the electron beam pulse width.

In order to detect a relative pulse-length change of the electron beam, we developed a waveguide spectrometer (WGSPM). This WGSPM allows us to monitor and tune the velocity-bunching process of the electron beam in the injector. Figure 18 shows the structure of the WGSPM, in which five waveguides with different cutoff wavelengths, $\lambda_{c}$, (i.e. having different $2 a$ values of the waveguides) are connected in series. The cutoff wavelengths ( $2 a$ sizes) are $0.08,0.04,0.02,0.01$, and $0.005 \mathrm{~m}$, respectively. This means that the successive waveguides are joined from a longer cutoff wavelength $(3.75 \mathrm{GHz})$ at an input aperture to the shortest one $(60 \mathrm{GHz})$ in a deeper space. Each waveguide has a $50 \mathrm{GHz} \mathrm{rf}$ detector connected with an electrical coupling antenna on the electrical surface of the

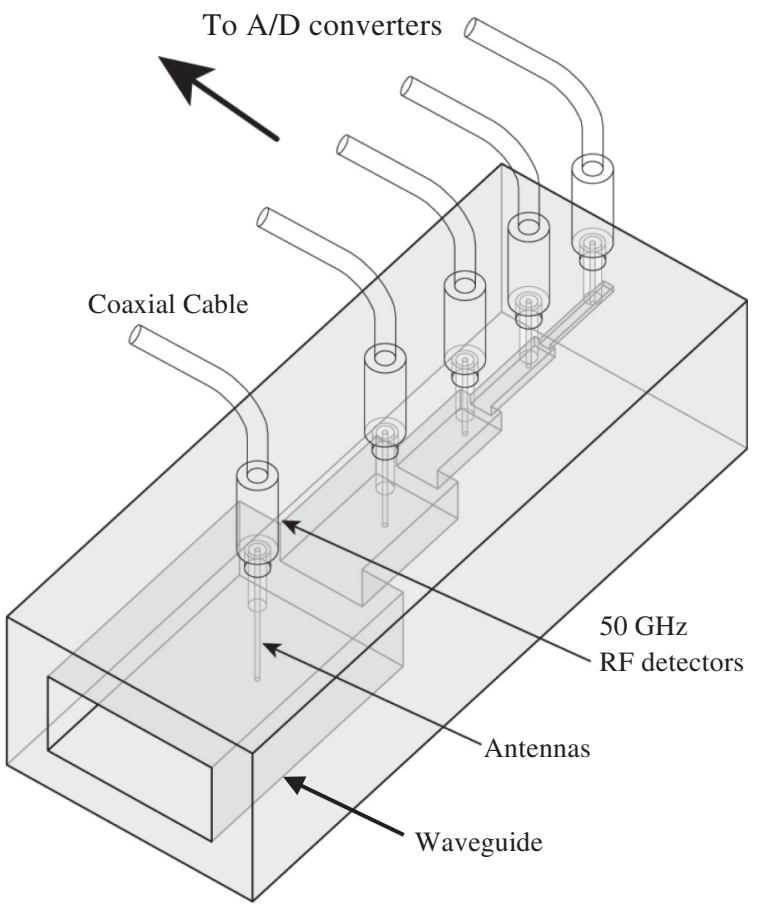

FIG. 18. Structure of the WGSPM comprising the five waveguides with the different cutoff frequencies. The individual waveguides have $50 \mathrm{GHz}$ rf detectors to observe the CTR.

waveguides. The transmission rf modes of the CTR signals are usually the TE and TM modes in the waveguide. Of course, the transmission modes are dependent on the polarization of a radiated CTR electromagnetic field. However, the CTR signal has a wide dynamic frequency range, because of a short pulse. When the wavelength of the CTR signal, $\lambda_{\text {sig }}$, is much shorter than the cutoff wavelength of the waveguide, a TEM-like mode is dominated in the waveguide due to the relation among $\lambda_{\text {sig }} \approx$ $\lambda_{g} \approx \lambda_{0} \ll \lambda_{c}$, where $\lambda_{g}$ is the guided wavelength in the waveguide and $\lambda_{0}$ is the wavelength in free space. Then this signal can be almost assumed as the TEM-like mode in free space, since the reflection angle of the transmission signal on a waveguide wall is very shallow. As a result, the antenna can couple the electrical field of these transmission modes.

When the CTR at the longer wavelength corresponding to the longer pulse width of the electron beam is input to the WGSPM, the rf detector at the first waveguide with the lower cutoff frequency can only detect the signal, because of a high-pass filter characteristic. Hence, the rf detectors attached at other waveguides following the first one cannot detect the CTR, because of the cutoff frequencies. If the CTR includes even much shorter wavelength components, the detectors at further deeper waveguides can observe the CTR. The further higher frequency signals with the TE or TEM modes than the cutoff frequency can easily transmit the connection discontinuity between the first and successive second waveguides without any strong reflections in 
the condition of $\lambda_{\text {sig }} \approx \lambda_{g} \approx \lambda_{0} \ll \lambda_{c}$. Therefore, the relative output intensity of the detector at each position depends on the input CTR spectrum. This fact to resolve the wavelengths by the waveguides with the different sizes is the reason why we call a waveguide spectrometer. Therefore this spectrometer can measure the relative bunch-length evolution governed by the velocity-bunching process in the injector.

Figure 19 depicts the output peak signals of the detectors, while the rf phase of the $476 \mathrm{MHz}$ booster cavity at the SCSS test accelerator was changed. These data clearly show the relative bunch-length dependence to the rf phase, which changes the acceleration voltage at the booster cavity in the velocity-bunching process. In the figure, the peak point of a solid line with a triangle in the case of the $15 \mathrm{GHz}$ cutoff frequency shows the shortest bunch length. The left side of the peak point is a bunching region and the right side of the peak is a debunching region. Therefore, the optimum bunching phase of the booster cavity in this case is at the slightly off-peak point to the bunching region. We can tune the bunching process of the injector part by this waveguide spectrometer.

\section{CSRM}

Since the fluctuation of the bunch length directly reflects the lasing intensity variation, the bunch-length measurement is crucial. The spectra of the coherent synchrotron radiation (CSR) include bunch-length information. Therefore, the intensity of coherent synchrotron radiation (CSR) [19] reflects a bunch-length change, as described blow. The CSR flux intensity, $P_{\mathrm{csr}}(f)$, is dependent on a frequency expressed by the equation [15]

$$
P_{\mathrm{csr}}(f) \sim P_{e}(f)\left[N_{e}+N_{e}^{2} F(f)\right],
$$

which is inversely proportional to the electron bunch length, $\tau$, because of $f \approx 1 / \tau$, where $N_{e}$ is the electron

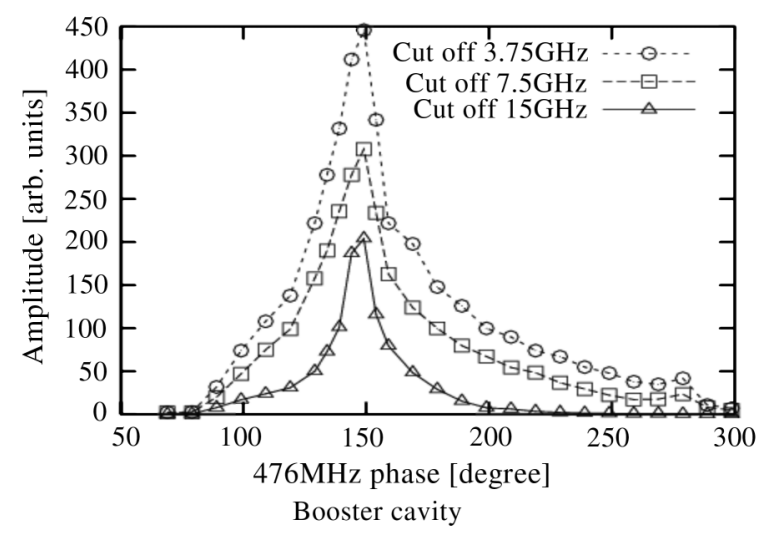

FIG. 19. Output voltage signals of the individual cutoff waveguides dependent on the rf phase of the $476 \mathrm{MHz}$ booster cavity at the SCSS test accelerator. The region below 150 degrees corresponds to an under bunching part and over 150 degrees corresponds to an over bunching part; 150 degrees is the rf crestacceleration point.

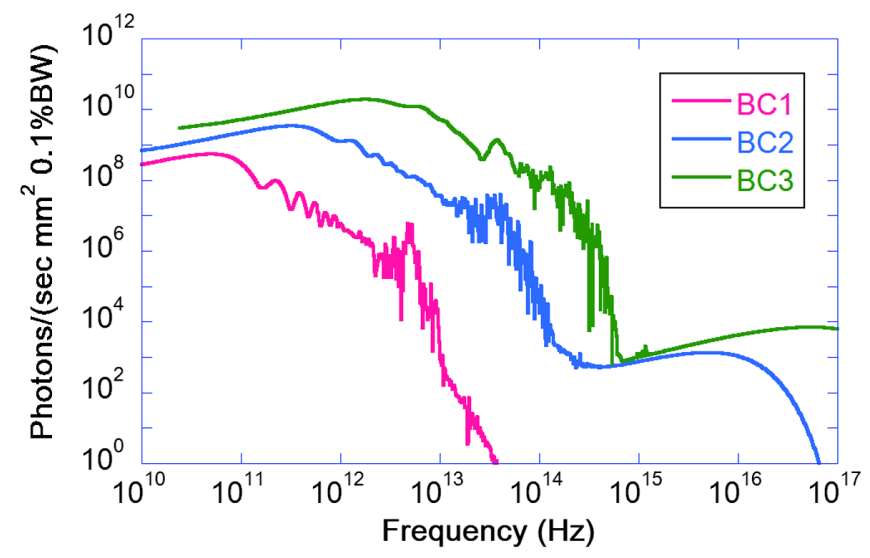

FIG. 20. Numerical calculations of the CSR intensities dependent on the wavelength at $\mathrm{BC} 1, \mathrm{BC} 2$, and $\mathrm{BC} 3$. Calculations clearly show that the CSR intensities increase, while the frequency increases.

population and $F(f)$ is the bunch form factor. The Gaussian distribution of the bunch shape is assumed to be the form factor. $P_{\mathrm{csr}}(f)$, which depends on the CSR frequencies at the individual $\mathrm{BC} 1-3$, is numerically calculated by SPECTRA [20]; its calculated results are plotted in Fig. 20.

A nondestructive bunch-length monitor, such as the CSRM used to observe the CSR, is very helpful to tune and control the electron beam bunch length from subpicosecond to several tens of femtoseconds. Because the spectra of the CSR signals have relative bunch-length information, as described in Eq. (6) and Fig. 20, we installed three nondestructive CSRMs, as shown in Fig. 21, at the three BCs, respectively. Each CSRM comprises a pyroelectric detector, an organic lens, a perforated and goldplated aluminum mirror, and a gold-plated mirror. They can observe CSRs emitted from the final bending magnets of the individual BCs comprising four bending magnets.

The fundamental frequencies of the CSRs based on the electron-bunch length at $\mathrm{BC} 1, \mathrm{BC} 2$, and $\mathrm{BC} 3$ are about $50 \mathrm{GHz}, 100 \mathrm{GHz}$, and $1 \mathrm{THz}$, respectively. The wavelengths at the peak CSR intensities vary in accordance with a change of the individual bunch lengths at the BCs. The frequency characteristic of the pyrodetector is like a

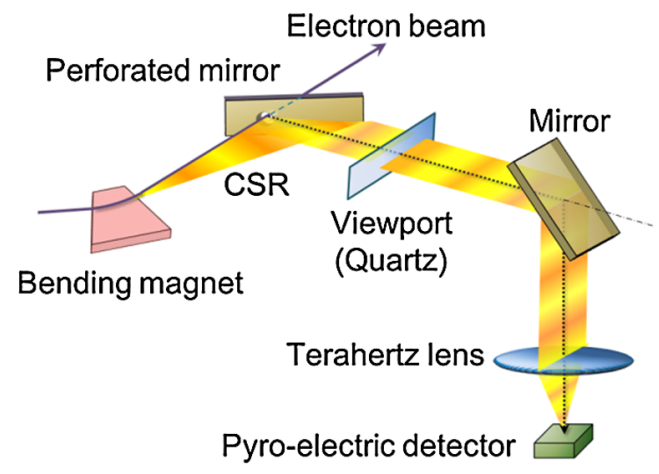

FIG. 21. Configuration of a typical CSR detection system. 


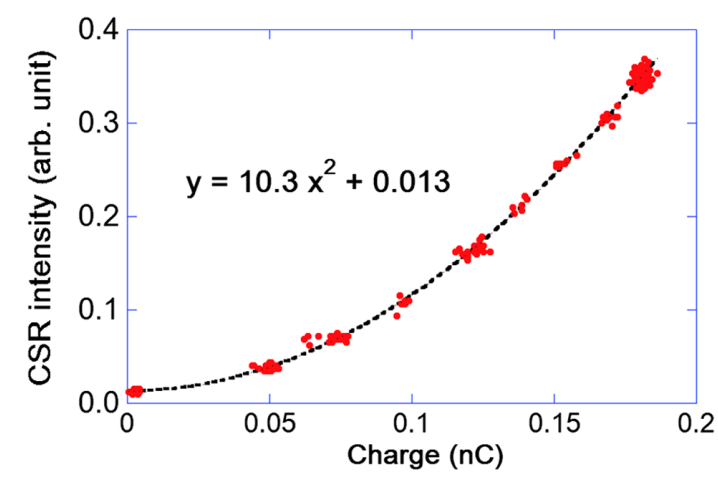

FIG. 22. Electron charge-amount dependence of the pyrodetector output. The intensity clearly changes in proportion to the square of the charge amount.

high-pass filter used to detect these frequency signals. Therefore, if the fundamental frequency components of the CSRM signal fit a flat place of the amplitude frequency characteristics of the detector at the individual BCs, the output voltages from the pyrodetectors also inversely change to be proportional to the bunch-length variation. Based on the principle expressed in Eq. (6) and the highpass filter characteristic, the CSRMs can detect relative variations of the electron bunch lengths.

Figure 22 depicts the electron charge-amount dependence of the pyrodetector output. This square proportionality characteristic in the figure is consistent with the calculation results of Eq. (6). Figure 23 shows the bunchlength sensitivity of the CSRM at $\mathrm{BC} 2$, when the energy chirp of the bunch was changed by trimming the rf phase of the S-band cavity before $\mathrm{BC} 2$. The graph in the figure shows good proportionality between the S-band cavity phase and the intensity of the CSRM output signal. This bunch-length measurement result is concordant with the fact that the energy chirp is converted into the electron bunch length through the $R 56$ value of each $\mathrm{BC}$.

\section{Streak camera system using OTR}

The OTR is generated when an electron beam hits on the mirror surface of a stainless-steel screen. This OTR is a

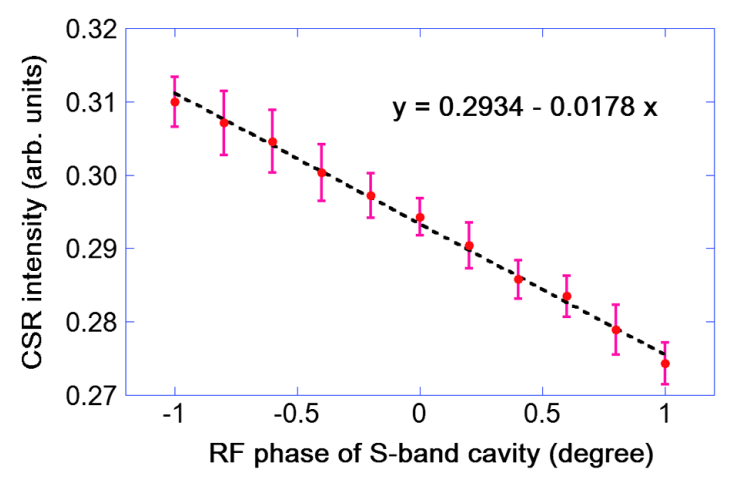

FIG. 23. CSR intensity variation at BC2, dependent on the rf phase change of the $\mathrm{S}$-band accelerating structure.

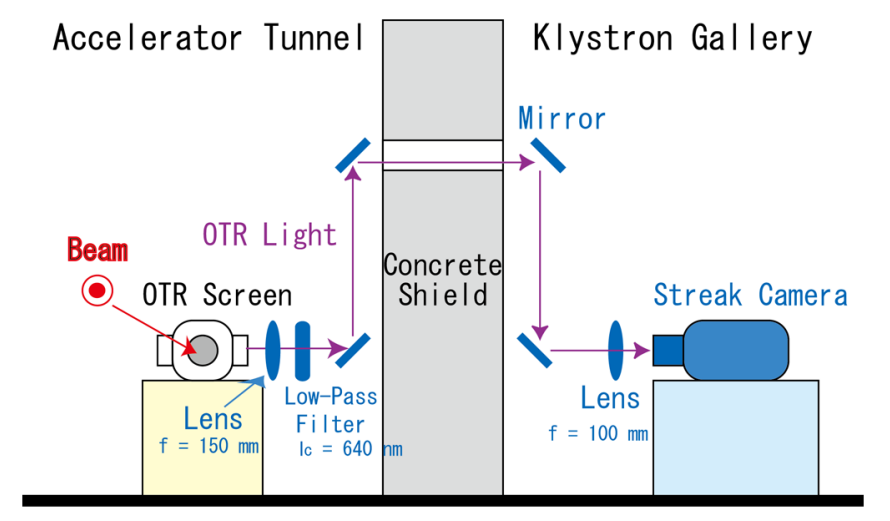

FIG. 24. Experimental setup of an electron bunch-length measurement using OTR light and a streak camera.

good probe to observe an electron bunch length of over several hundred femtoseconds, because of the fast time response. A streak camera, which allows us to observe the OTR with a pulse width of more than $300 \mathrm{fs}$, is a suitable device for a fast temporal phenomenon. Hence, we employed a streak camera of FESCA 200 (Hamamatsu Photonics) to measure electron bunch lengths from $1 \mathrm{ps}$ to 300 fs by using the SCMs with the OTR screens for the accelerator part upstream of $\mathrm{BC} 2$.

Figure 24 is an illustration of the installed bunch-length measurement system using a streak camera, an optical system, and the SCM. The SCM is placed just after the chicane of BC3, as shown in Fig. 1. The OTR emitted from the SCM is guided along the optical system using mirrors, and is fed into the streak camera. To reduce any effect due to wavelength-dependent optical group delays along the optical path, and energy dispersion on the photoelectric surface of the streak camera, an optical low-pass filter and a reflection optical-geometrical system are employed. Because the temporal resolution of this system deteriorates due to the optical-dispersion effect dependent on the wavelength, a cutoff wavelength, $\lambda_{c}$, of the filter of $640 \mathrm{~nm}$ is chosen. The wavelength sensitivity of the streak camera is limited to be shorter than about $850 \mathrm{~nm}$, like a high-pass filter (of course, there is the shortest limit wavelength of the sensitivity of the camera). Therefore, the wavelength sensitivity of this bunch-length measurement system is from 850 to $640 \mathrm{~nm}$, like a band-pass filter. To obtain sufficient optical intensity, which reflects an improvement of the signal-to-noise $(\mathrm{S} / \mathrm{N})$ ratio, a focusing lens placed near the streak camera is employed. Figure 25 shows the results of electron bunch-length measurements by using the steak camera. The bunch-length wave form in the figure is made by stacking ten single-shot wave forms, which is adjusted at each peak value timing. The bunch length observed with the streak camera is about $300 \mathrm{fs}$ (FWHM).

\section{RFDEF}

At the present time, an RFDEF, as a sort of streak camera, is only an instrument used to observe an electron 


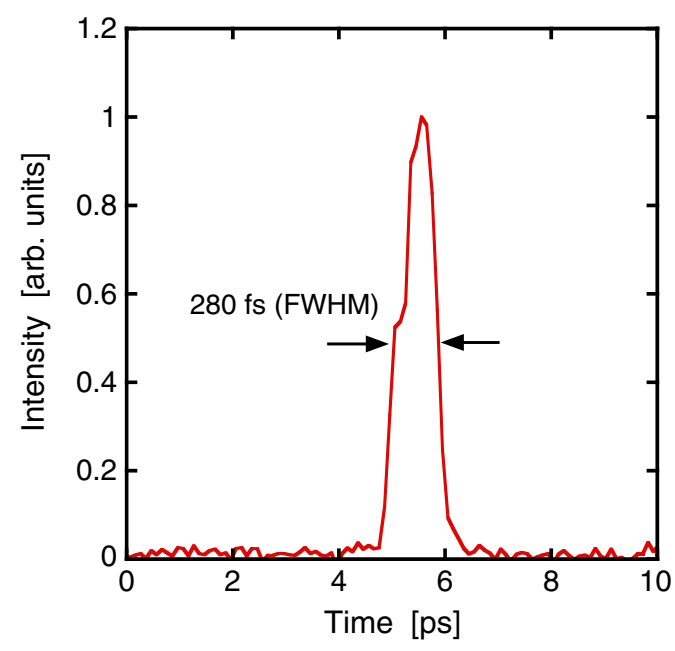

FIG. 25. Observed bunch-shape taken with the streak camera using the OTR. The bunch wave form is reconstructed by stacking ten single-shot wave forms while adjusting each peak value timing. The bunch length is about $300 \mathrm{fs}$ in FWHM.

bunch length of below $300 \mathrm{fs}$. Hence, we employed the RFDEF to diagnose a $30 \mathrm{fs}$ electron bunch of SACLA. The bunch-length measurement system, as shown in Fig. 26, comprises a C-band rf deflector (RFDEF), a beam drift space, and a high spatial resolution SCM, as mentioned in the previous section. This RFDEF operated at $5712 \mathrm{MHz}$ is driven by a high-power rf source including a $50 \mathrm{MW}$ pulse klystron.

The operating principle of the RFDEF is as follows. The RFDEF is a backward traveling-wave structure at the operation rf mode of HEM11. When the electron beam is injected into the structure at an rf zero-cross phase, the RFDEF pitches the beam bunch around its center to project an image of a longitudinal bunch structure on the screen of the above-mentioned SCM. The relation between the deflection voltage, $V_{y}$, and the projected bunch length on the screen, $l_{y}$, is given by

$$
V_{y}=\frac{l_{y}}{L_{d}} \frac{c p_{z}}{e k_{a} \sigma_{z}}
$$

where $L_{d}$ is the drift length between the RFDEF longitudinal center and the surface of the SCM screen, $k_{a}$ is the wave number of the RFDEF, $\sigma_{z}$ is the bunch length, and $p_{z}$ is the longitudinal momentum of the electron bunch. $V_{y}$ must be $40 \mathrm{MV}$ in the case of $L_{d}=5 \mathrm{~m}$

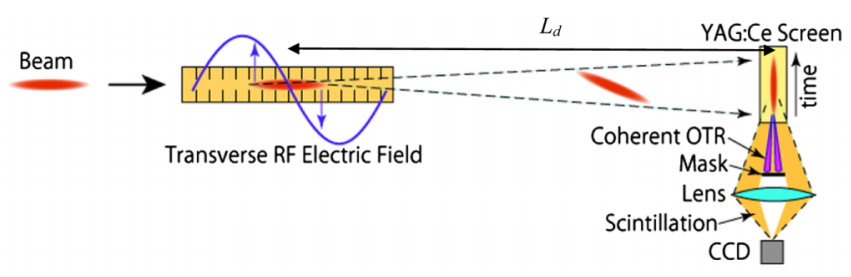

FIG. 26. Bunch-length measurement system using the RFDEF to observe a pulse width of less than $500 \mathrm{fs}$.

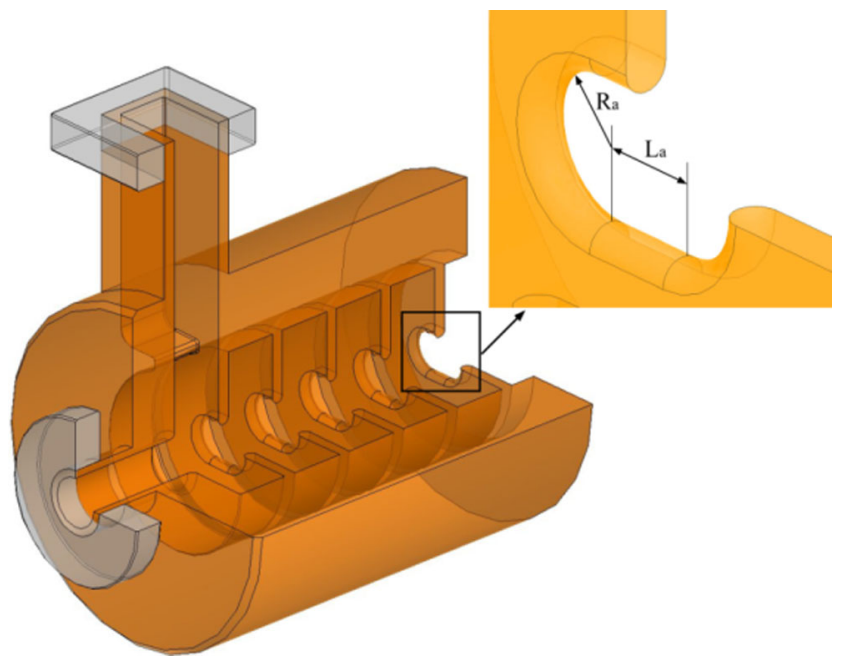

FIG. 27. HEM-11-(5/6) $\pi$-mode backward-traveling-wave accelerating structure with racetrack-shape rf coupling irises to prevent $X$ - and $Y$-mode mixing.

to obtain a bunch-length measurement sensitivity of $200 \mathrm{fs} / \mathrm{mm}$ on the screen of the SCM with a spatial resolution of less than $2.5 \mu \mathrm{m}$.

To realize this measurement system, a special backward traveling-wave accelerating structure with the HEM11-(5/6) $\pi$ transverse mode at $5712 \mathrm{MHz}$ was developed, as shown in Fig. 27. This accelerating structure has racetrack-shape rf coupling irises to prevent rotation of the deflection plane of the HEM11 mode. The main parameters of this backward traveling-wave accelerating structure are tabulated in Table II, and the dispersion relations of the $X$ and $Y$ modes in a fabrication model of the accelerating structure are depicted in Fig. 28. In the figure, the $X$ and $Y$ modes are sufficiently separated by the iris. Furthermore, even though the (5/6) $\pi$ mode is employed, a group velocity, $v_{g}$, of about $0.02 c$ in the accelerating structure is achieved. These rf characteristics guarantee stable highpower rf operation of the accelerating structure.

TABLE II. Rf specifications of the RFDEF.

\begin{tabular}{lccc}
\hline \hline Total deflecting voltage & $V_{y}$ & 40 & $\mathrm{MV}$ \\
rf deflecting phase & $\phi_{a}$ & 0 & degree \\
Fractional bunch length for & $\sigma_{z}$ & 200 & $\mathrm{fs}$ \\
$\quad$ x-ray oscillation & & & \\
Beam energy at the deflector & $p_{z} c$ & 1.45 & $\mathrm{GeV}$ \\
Resonant frequency & $f_{a}$ & 5712 & $\mathrm{MHz}$ \\
Type of structure & & $\mathrm{CZ}$ & \\
Resonant mode & & $\mathrm{HEM} 11$ & \\
Phase shift per cell & $\beta D$ & $5 \pi / 6$ & $\mathrm{rad}$ \\
Group velocity & $v_{g} / c$ & -2.16 & $\%$ \\
Filling time & $T_{f}$ & 0.27 & $\mu \mathrm{s}$ \\
Unloaded $Q$ & $Q_{a}$ & 11500 & \\
Transverse shunt impedance & $z_{y}$ & 13.9 & $\mathrm{M} \Omega / \mathrm{m}$ \\
Length of structure & $L$ & $1.7 \times 2$ & $\mathrm{~m}$ \\
\hline \hline
\end{tabular}




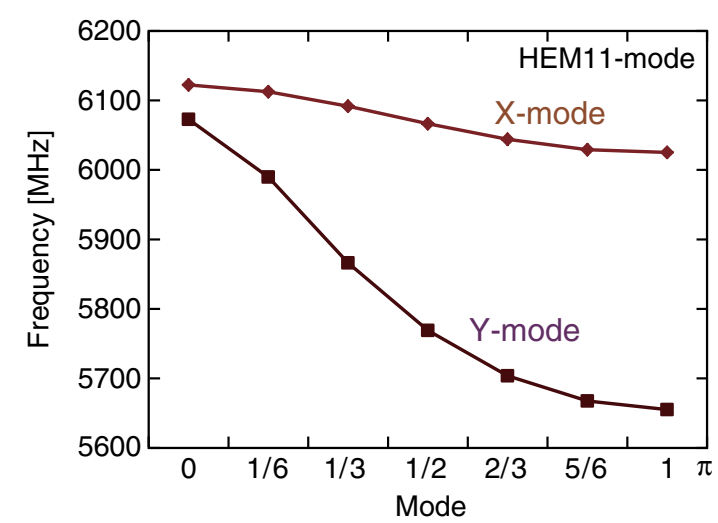

FIG. 28. Measured dispersion relations of the $X$ and $Y$ HEM-11 modes.

This system was installed into the accelerator tunnel of SACLA. The two RFDEFs are used and driven by a high-power rf source including a $50 \mathrm{MW}$ pulse klystron. After rf conditioning for the RFDEFs, a $60 \mathrm{MV}$ beam pitching voltage was obtained. We tested this system performance by using SACLA electron beams, and finally obtained a beam deflection image on the SCM, as depicted in Fig. 29(a). Figure 29(a) shows images before (A) and after (B) activating the RFDEF, respectively. The temporal wave form of the beam current in Fig. 29(a), (B), is shown in Fig. 29(b). The peak current is $2.75 \mathrm{kA}$ with its pulse width of $40 \mathrm{fs}$, which is almost consistent with the designed value of the compressed beam peak current. A calibration of the temporal resolution of this system, as shown in Fig. 30, was also carried out. For this calibration, the movement of the center-mass position of the pitched beam image on the screen was plotted by shifting the input rf phase of the RFDEF from the klystron, while the deflection voltage of the RFDEF was set to $22 \mathrm{MV}$. The effective resolution of the bunch-length measurement is about $20 \mathrm{fs}$. This value is mainly limited by the natural beam size, being determined due to a beta function $(60 \mathrm{~m})$ and a natural beam emittance (a normalized emittance is $1.1 \pi \mathrm{mm} \mathrm{mrad})$.
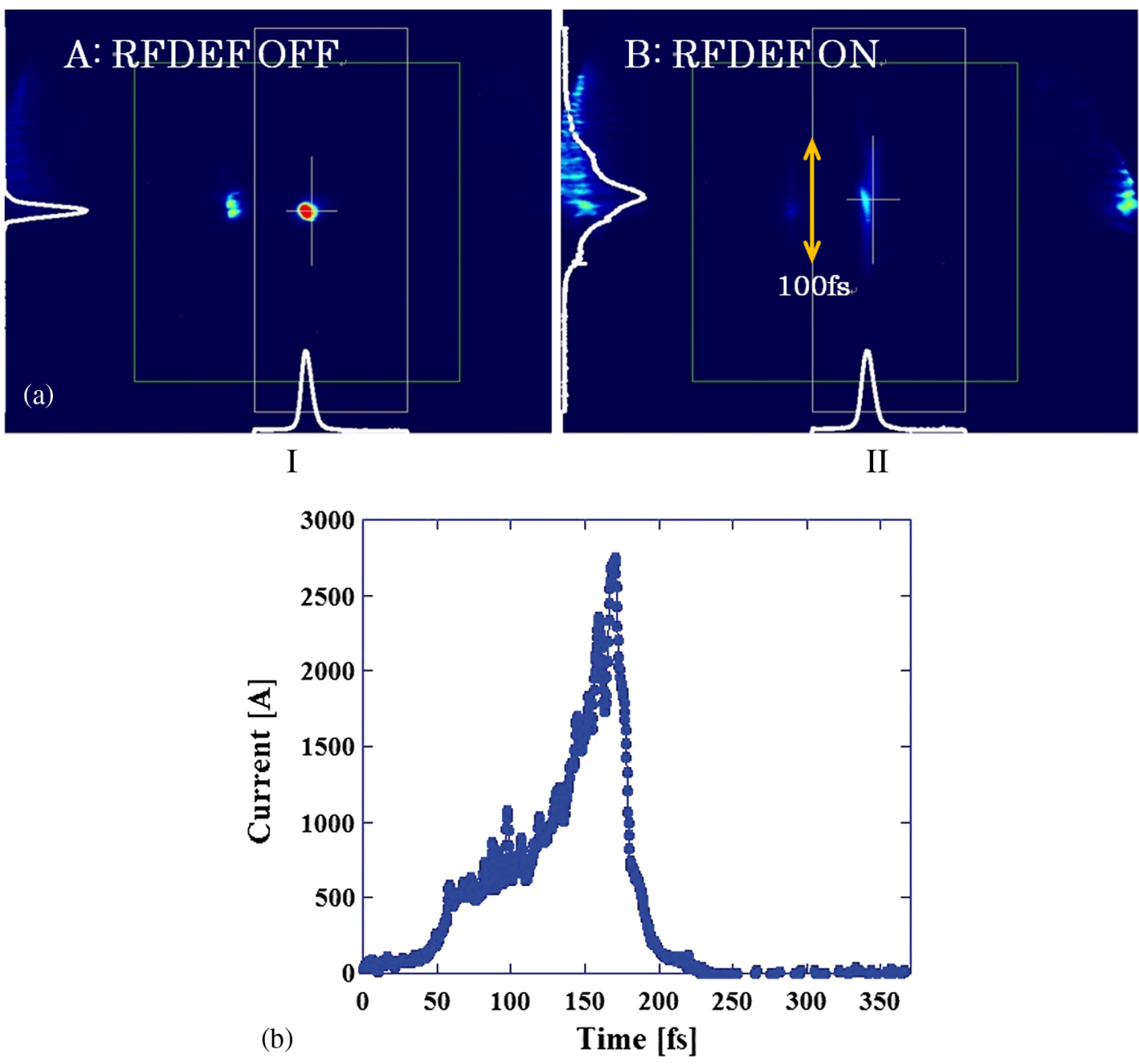

FIG. 29. (a) A, the beam profile (left) taken with the Ce:YAG screen; B, the beam image (right) pitched by the RFDEF and taken with the same screen. (b) Temporal beam-current wave form in Fig. 29(a) taken by the RFDEF system. The bunch length in this case is about 40 fs in FWHM. 


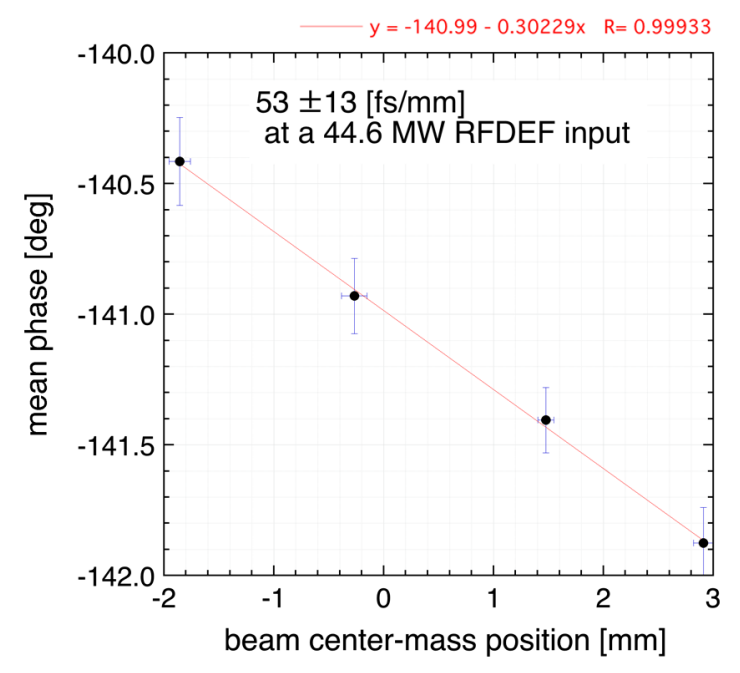

FIG. 30. Calibration of the temporal resolution of the bunchlength measurement system using the RFDEF. The calibration factor of the temporal resolution of the RFDEF is $53 \pm$ $13 \mathrm{fs} / \mathrm{mm}$. The error bars are STD.

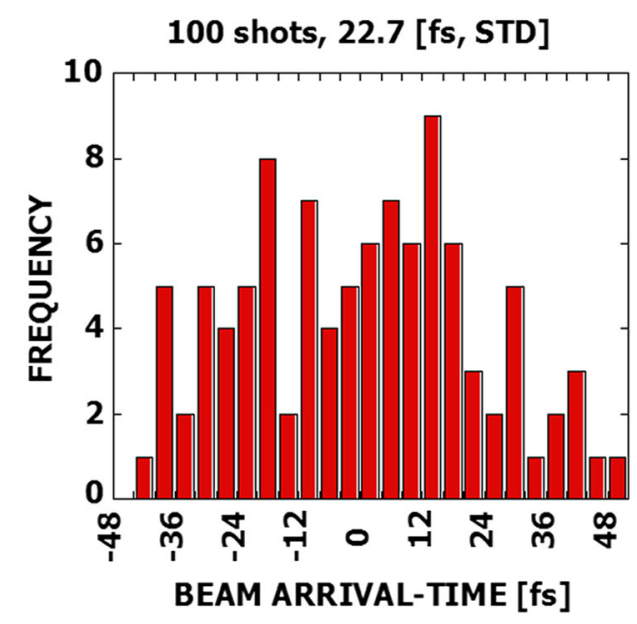

FIG. 31. Beam arrival time (movement of the center-mass position of the beam image) measured by the rf deflector system. The arrival-time jitter is $22.7 \mathrm{fs}$ in STD.

We also measured the beam arrival-time jitter with the RFDEFs, and show its results in Fig. 31. In this case, the beam arrival time jitter was $22.7 \mathrm{fs}$ in standard deviation (STD) with the conditions that the beam energy was $1.45 \mathrm{GeV}$, the beam charge was about $0.3 \mathrm{nC}$, and the beam repetition cycle was 1 pps. This temporal resolution of the jitter is also limited by the natural beam size (emittance). This measurement accuracy is strongly supported by the rf phase and the amplitude stabilities of our low-level rf system for SACLA [21,22].

\section{EVALUATION OF THE BEAM MONITORS}

\section{A. Spatial cross evaluation between the BPM and SCM}

In order to establish further reliability of the spatial resolutions of the BPM and SCM, as mentioned previously,

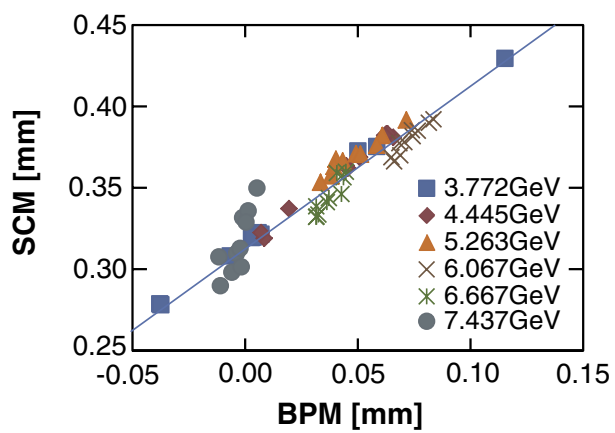

FIG. 32. Correlation plots between the center-mass positions taken with the BPM and SCM of BL3-2, while the electron beam energy was changed. Different energy data are indicated by the different color and shaped dots. The solid line shows the first order fitted data. Both the position data of the BPM and SCM have a good linear relation, and correlate within $10 \mu \mathrm{m}$ in rms.

a cross evaluation of them was carried out. This evaluation was made by correlation measurements of the beam centerof-mass positions along an $\mathrm{x}$ axis with both the BPM and SCM in BL3-2, which are installed nearby the undulator. In the case of the SCM, the $\mathrm{x}$-axis center-mass position was calculated from image data taken with it. Figure 32 shows correlation plots between these data of the center mass positions, while the electron beam energy was changed. Both position data still have a good linear relation between them, and correlates within $10 \mu \mathrm{m}$ in rms. Even though the beam profiles were different at the individual beam energies, their correlations were maintained. This fact is particularly notable.

\section{B. Temporal resolution evaluation among the monitors}

A cross evaluation of temporal resolutions of the monitors is very important to make sure of their reliability. Thus, the electron beam bunch lengths with almost the same conditions were measured with a streak camera

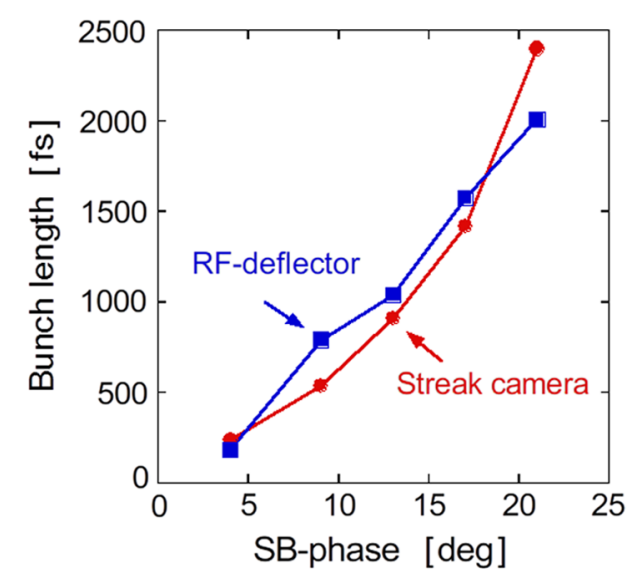

FIG. 33. Correlation data of the bunch-length variation measured with a streak camera and the rf deflector. 


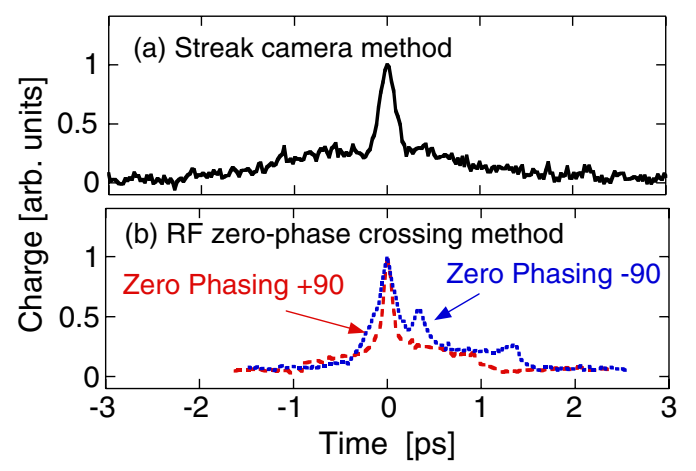

FIG. 34. Cross-check data among the electron bunch lengths measured with the streak camera (black solid line) and the rf zero-crossing methods (red and blue dotted lines). + 90 degrees is an acceleration phase condition in the C-band traveling accelerating structures before the bunch compressor and -90 degrees is a deceleration phase condition. Both of the measured bunch lengths are about $300 \mathrm{fs}$ in FWHM.

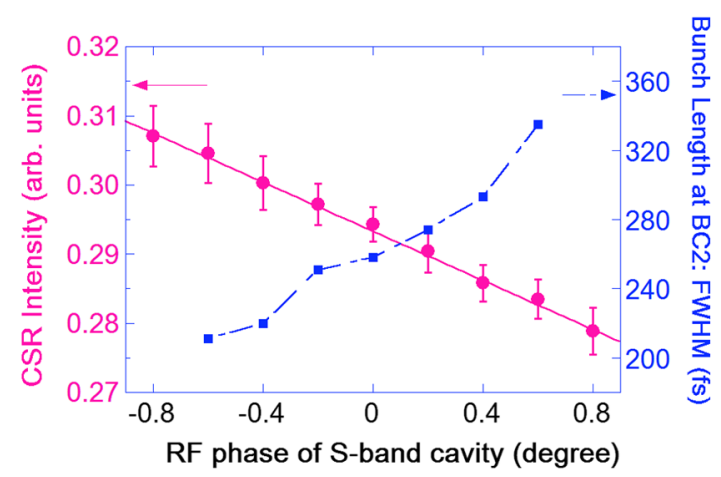

FIG. 35. Performance comparison between the CSRM and the RFDEF by using both of the observed bunch-length data. The dotted blue line with the solid squares indicates the bunch-length data (right vertical axis) taken by the RFDEF. The red solid line with solid circles expresses the intensity data of the CSRM signal (left vertical axis) from the pyrodetector at the BC2, which reflects the relative bunch length.

observing the OTR and the RFDEF. The bunch-length data measured with the streak camera were also compared with other schemes, such as the rf zero-crossing method [23] at the SCSS test accelerator [24].
Figure 33 shows correlation data of the bunch-length variation measured with the streak camera and the RFDEF. The vertical axis of the figure is a bunch length measured with the RFDEF and the streak camera. The horizontal axis is the rf phase of the S-band accelerating structures before $\mathrm{BC} 2$ to change the bunch length. They have a good correlation with a factor of about 1 .

The cross-check data among the streak camera and rf zero crossing are depicted in Fig. 34. In the case of the rf zero crossing, two cases of +90 degrees and -90 degrees described in the figure were measured. These conditions correspond to an acceleration phase of +90 degrees and a deceleration phase of -90 degrees in the C-band accelerating structure before the energy dispersion section of the bunch compressor. Details of the experimental methods are given in Ref. [24]. The measured bunch-length data taken with them are almost consistent. The bunch lengths were about $300 \mathrm{fs}$ in FWHM.

A performance comparison between the CSRM at $\mathrm{BC} 2$ and the RFDEF was also made. Figure 35 shows the bunch-length variations measured by them, while the rf phase of the S-band accelerating structure varied. Both the measured data of the bunch-length change are almost linear, which proves their measurement reliability. In this case, the CSRM signal intensities on the graph reflect the electron bunch lengths, and the data taken with the RFDEF are the exact electron bunch length.

\section{Performance summary of the monitors}

Above-mentioned beam monitors show sufficient performance for the beam tuning of SACLA. Table III tabulates the achieved spatial and temporal resolutions of the individual monitors, as well as their design target resolutions.

\section{SUMMARY}

The developed beam monitors for SACLA have worked well. They greatly contribute to beam tuning, such as by

TABLE III. Achieved spatial and temporal resolutions of the individual beam monitors and their design resolutions.

\begin{tabular}{lcc}
\hline \hline Instrument & Designed target resolution & Achieved resolution \\
\hline Spatial observation & $<1 \mu \mathrm{m}$ in rms & $0.6 \mu \mathrm{m}$ in rms \\
BPM & $<3 \mu \mathrm{m}$ in FWHM & $2.5 \mu \mathrm{m}$ in FWHM \\
SCM & $<500 \mathrm{ps}$ in $10 \%-90 \%$ rise time & \\
Temporal observation & $1 \mathrm{~ns}-10 \mathrm{ps}$ in injector & $200 \mathrm{ps} \mathrm{in} 10 \%-90 \%$ rise time \\
DCT & $1 \mathrm{ps}-30 \mathrm{fs}$ at BC1-3 & $1 \mathrm{~ns}-10 \mathrm{ps}$ \\
WGSPM & $<300 \mathrm{fs}$ at BC2 & $1 \mathrm{ps}-30 \mathrm{fs}$ \\
CSRM & $<30 \mathrm{fs}$ at BC3 & $300 \mathrm{fs}$ \\
Streak camera with OTR & $23 \mathrm{fs}$ \\
RFDEF & & \\
\hline \hline
\end{tabular}


adjusting the bunch length during velocity and magnetic bunching processes in the injector and BCs, respectively. For spatial observations, a BPM exhibited a position resolution of $0.6 \mu \mathrm{m}(\mathrm{rms})$, and the SCM has less than a $3 \mu \mathrm{m}$ (rms) spatial resolution. Owing to this spatial resolution of the SCM, the emittance value of an electron beam can be measured up to $1.1 \pi \mathrm{mm}$ mrad. For temporal observations, a DCT, a WGSPM, a CSRM, and an RFDEF show the demanded temporal resolutions, such as a bunchlength measurement resolution of about 20 fs observed with the RFDEF. These resolutions are strongly supported by the firm and accurate mechanical structures of the monitor bodies and a low-noise DAQ system. To check the reliability of our developed beam monitors, cross evaluations of them in both spatial and temporal dimensions were carried out. These cross-evaluated data were consistent with each other, and these evaluations secure the reliability of the above-mentioned resolutions of the monitors. Since we have realized sufficient resolutions of our developed beam monitors, the electron beam preserving its emittance of about $1 \pi \mathrm{mm}$ mrad is transported from the electron gun to after BC3. Hence, SACLA was successfully lased at $0.12 \mathrm{~nm}$. This fruitful result was strongly supported by our developed beam-monitor system for SACLA.

\section{ACKNOWLEDGMENTS}

We thank the members of the XFEL/SPring- 8 construction group for their support to our study and development.

[1] T. Ishikawa et al., Nat. Photonics 6, 540 (2012).

[2] Jianwei. Miao, Pambos Charalambous, Janos Kirz, and David Sayre, Nature (London) 400, 342 (1999).

[3] E. L. Saldin, E. A. Schneidmiller, and M. V. Yurkov, The Physics of Free Electron Laser (Springer, London, 2000), p. 52.

[4] SCSS X-FEL Conceptual Design Report, edited by T. Tanaka and T. Shintake (RIKEN Harima Institute, Japan, 2005).

[5] S. Hiramatsu, KEK OHO'98 High-Energy Accelerator Seminar (Foundation for High Energy Accelerator Science, Tsukuba, 1998), pp. I-1-I-55, in Japanese.

[6] T. Tanaka, H. Kitamura, and T. Shintake, Nucl. Instrum. Methods Phys. Res., Sect. A 528, 172 (2004).

[7] S. Y. Lee, Accelerator Physicis (World Scientific, Singapore, 2007), 2nd ed., p. 62.

[8] H. Hanaki, T. Asaka, H. Ego, H. Kimura, T. Kobayashi, S. Suzuki, T. Fukui, T. Inagaki, N. Kumagai, Y. Otake,
T. Shintake, K. Togawa, and M. Yamaga, in Proceedings of the 25th International Linear Accelerator Conference LINAC10, Tsukuba, Japan (KEK, Tsukuba, Japan, 2010), p. 425.

[9] H. Tanaka, T. Hara, and K. Togawa, in Proceedings of the 4th Annual Meeting of PASJ (Particle Accelerator Society of Japan, Tokyo, 2007), p. 613, in Japanese.

[10] H. Maesaka, H. Ego, S. Inoue, S. Matsubara, T. Ohshima, T. Shinatake, and Y. Otake, Nucl. Instrum. Methods Phys. Res., Sect. A 696, 66 (2012).

[11] K. Yanagida, H. Tomizawa, A. Yamashita, S. Inoue, and Y. Otake, in Proceedings of the 24th International Linear Accelerator Conference LINAC08 (2008), p. 573.

[12] J.D. Jakson, Classical Electrodynamics (John Wiley \& Sons, New York, 1975), 2nd ed., p. 685.

[13] S. Matsubara, K. Yanagida, H. Maesaka, H. Ego, A. Higashiya. Y. Otake, and S. Inoue, in Proceedings of IPAC2011, San Sebastián, Spain (EPS-AG, Spain, 2011), p. 1227.

[14] H. Maesaka, T. Shintake, Y. Otake, T. Hara, K. Togawa, T. Tanikawa, M. Yabashi, H. Tanaka et al., in Proceedings of the 3rd Annual Meeting of Particle Accelerator Society of Japan (Particle Accelerator Society of Japan, Tokyo, 2006), p. 328, in Japanese.

[15] C. Kondo, H. Maesaka, Y. Otake, S. Matsubara, T. Matsumoto, and S. Inoue, in Proceedings of IPAC2011, San Sebastián, Spain (Ref. [13]), p. 1224.

[16] H. Ego, H. Maesaka, Y. Otake, T. Sakauri, S. Miura, and T. Hashirano, in Proceedings of IPAC2011, San Sebastián, Spain (Ref. [13]), p. 1221.

[17] Espace Allondon Ouest, 156, rue du Mont Rond 01630 Saint Genis Pouilly, France.

[18] U. Happek, A. J. Sievers, and E. B. Blum, Phys. Rev. Lett. 67, 2962 (1991).

[19] T. Nakazato, M. Oyamada, N. Niimura, S. Urasawa, O. Konno, A. Kagaya, R. kato, T. Kamiyama, Y. Torizuka, T, Nanba, Y. Kondo, Y. Shibata, K. Ishi, T. Ohsaka, and M. Ikezawa, Phys. Rev. Lett. 63, 1245 (1989).

[20] T. Tanaka and H. Kitamura, J. Synchrotron Radiat. 8, 1221 (2001).

[21] Y. Otake, T. Ohshima, N. Hosoda, H. Maesaka, T. Fukui, T. Ohata, M. Musya, K. Tamasaku, M. Kitamura, K. Imai, M. Kourogi, and T. Shinatake, in Proceedings of the ICALEPCS07 (2007), p. 706.

[22] Y. Otake, T. Ohshima, N. Hosoda, H. Maesaka, T. Fukui, M. Kitamura, and T. Shinatake, Nucl. Instrum. Methods Phys. Res., Sect. A 696, 151 (2012).

[23] D. X. Wang, G. A. Krafft, and C. K. Sinclair, Phys. Rev. E 57, 2283 (1998).

[24] Y. Otake, S. Matsubara, H. Maesaka, K. Tamasaku, T. Togashi, K. Togawa, H. Tanaka, and M. Goto, in Proceedings of the 14th Beam Instrumentation Workshop, Santa Fe, New Mexico (LANL, Los Alamos, 2010), p. 307. 